\title{
The Unipotent Albanese Map and Selmer Varieties for Curves
}

\author{
Dedicated to the memory of my teacher Serge Lang
}

By

Minhyong KIM*

\begin{abstract}
We study the unipotent Albanese map that associates the torsor of paths for p-adic fundamental groups to a point on a hyperbolic curve. It is shown that the map is very transcendental in nature, while standard conjectures about the structure of mixed motives provide control over the image of the map. As a consequence, conjectures of 'Birch and Swinnerton-Dyer type' are connected to finiteness theorems of Faltings-Siegel type.
\end{abstract}

In a letter to Faltings [16] dated June, 1983, Grothendieck proposed several striking conjectural connections between the arithmetic geometry of 'anabelian schemes' and their fundamental groups, among which one finds issues of considerable interest to classical Diophantine geometers. Here we will trouble the reader with a careful formulation of just one of them. Let $F$ be a number field and

$$
f: X \rightarrow \operatorname{Spec}(F)
$$

a smooth, compact, hyperbolic curve over $F$. After the choice of an algebraic closure

$$
y: \operatorname{Spec}(\bar{F}) \rightarrow \operatorname{Spec}(F)
$$

and a base point

$$
x: \operatorname{Spec}(\bar{F}) \rightarrow X
$$

Communicated by A. Tamagawa. Received June 11, 2007. Revised December 21, 2007. 2000 Mathematics Subject Classification(s): 14G05, 11G30.

* Department of Mathematics, University College London, Gower Street, London, WC1E 6BT, United Kingdom, and The Korea Institute for Advanced Study, Hoegiro 87, Dongdaemun-Gu, Seoul 130-722, Korea.

e-mail: minhyong.kim@ucl.ac.uk

(c) 2009 Research Institute for Mathematical Sciences, Kyoto University. All rights reserved. 
such that $f(x)=y$, we get an exact sequence of fundamental groups:

$$
0 \rightarrow \hat{\pi}_{1}(\bar{X}, x) \rightarrow \hat{\pi}_{1}(X, x) \stackrel{f_{*}}{\rightarrow} \Gamma \rightarrow 0,
$$

where $\Gamma=\operatorname{Gal}(\bar{F} / F)$ is the Galois group of $\bar{F}$ over $F$ and $\bar{X}=X \otimes \bar{F}$ is the base change of $X$ to $\bar{F}$. Now, suppose we are given a section $s \in X(F)$ of the map $X \rightarrow \operatorname{Spec}(F)$, i.e., an $F$-rational point of $X$. This induces a map of fundamental groups

$$
s_{*}: \Gamma \rightarrow \hat{\pi}_{1}\left(X, x^{\prime}\right)
$$

where $x^{\prime}=s(y)$. Choosing an étale path $p$ from $x^{\prime}$ to $x$ determines an isomorphism

$$
c_{p}: \hat{\pi}_{1}\left(X, x^{\prime}\right) \simeq \hat{\pi}_{1}(X, x), \quad l \mapsto p \circ l \circ p^{-1},
$$

which is independent of $p$ up to conjugacy. On the other hand, $p$ maps to an element $\gamma \in \Gamma$, and it is straightforward to check that $c_{p} \circ s_{*} \circ c_{\gamma^{-1}}$ is a continuous splitting of $f_{*}: \hat{\pi}_{1}(X, x) \rightarrow \Gamma$. This splitting is well-defined up to the equivalence relation $\sim$ given by conjugacy of sections, where $\gamma \in \hat{\pi}_{1}(X, x)$ acts on a section $s$ as $s \mapsto c_{\gamma} \circ s \circ c_{f_{*}(\gamma)^{-1}}$. We get thereby a map

$$
s \mapsto\left[c_{p} \circ s_{*} \circ c_{\gamma^{-1}}\right]
$$

from $X(F)$ to the set $\operatorname{Split}(X)) / \sim$ of splittings $\operatorname{Split}(X)$ of the exact sequence modulo the equivalence relation $\sim$ given by conjugation. Grothendieck's section conjecture states that this map is a bijection:

$$
X(F) \simeq \operatorname{Split}(X) / \sim
$$

It seems that during the initial period of consideration, there was an expectation that the section conjecture would 'directly imply' the Mordell conjecture. At present the status of such an implication is unclear. Nevertheless, this fact does not diminish the conceptual importance of the section conjecture and its potential for broad ramifications in Diophantine geometry. In the long run, one can hope that establishing the correct link between Diophantine geometry and homotopy theory will provide us with the framework for a deeper understanding of Diophantine finiteness, especially in relation to the analytic phenomenon of hyperbolicity.

In this paper, we wish to make some preliminary comments on the fundamental groups/Diophantine geometry connection from a somewhat different perspective which, needless to say, does not approach the depth of the section conjecture. In fact, in our investigation, the main tool is the motivic fundamental group, especially $p$-adic realizations, rather than the pro-finite fundamental 
group. Nevertheless, what emerges is a (murky) picture containing at least a few intriguing points of mystery that are rather surprising in view of the relative poverty of unipotent completions. That is to say, one is surprised by the Diophantine depth of an invariant that is so close to being linear. It is the author's belief that the Selmer varieties arising in this context, generalizing $\mathbb{Q}_{p^{-}}$ Selmer groups of abelian varieties, are objects of central interest. Developing the formalism for a systematic study of Selmer varieties is likely to be crucial for continuing research along the lines suggested in this paper. However, the nonabelian nature of the construction presents a formidable collection of obstacles that are at present beyond the author's power to surmount. In spite of this, it is hoped that even a partial resolution of the problems can point us eventually towards the full algebraic completion of the fundamental group, bringing a sort of 'motivic Simpson theory' to bear upon the study of Diophantine sets.

We proceed then to a brief summary of the notions to be discussed and a statement of the results, omitting precise definitions for the purposes of this introduction. Although it will be clear that at least part of the formalism is more general, we will focus our attention on curves in this paper, right at the outset. So we let $F$ be a number field and $X / F$ a smooth hyperbolic curve, possibly non-compact. Let $R$ be the ring of $S$-integers in $F$ for some finite set $S$ of primes. We assume that we are given a smooth model

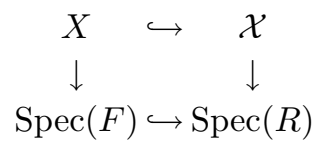

of $X$ and a compactification $\mathcal{X} \hookrightarrow \mathcal{X}^{\prime}$ relative to $R$ where $\mathcal{X}^{\prime}$ and the complement $\mathcal{D}$ of $\mathcal{X}$ in $\mathcal{X}^{\prime}$ are also smooth over $R$. The Diophantine set of interest in this situation is $\mathcal{X}(R)$, the $S$-integral points of $\mathcal{X}$. The theorems of Siegel and Faltings say that this set is finite. After setting up the preliminary formalism, our goal will be to investigate these theorems from a $\pi_{1}$-viewpoint.

As already mentioned, the $\pi_{1}$ we will be focusing on in this paper is a part of the motivic $\pi_{1}$ defined by Deligne [10]. In spite of the terminology, we do not rely on any general theory of motives in our discussion of the fundamental group. Rather, there will always be specific realizations related by the standard collection of comparison maps. Nevertheless, it is probably worth emphasizing that the main idea underlying our approach is that of a motivic unipotent Albanese map, of which the one defined by Hain [17] is the Hodge realization. That is, whenever a point $b \in \mathcal{X}(R)$ is chosen as a basepoint, a unipotent motivic fundamental group $U^{m o t}:=\pi_{1}^{m o t}(X, b)$ as well as motivic torsors $P^{m o t}(y):=\pi_{1}^{m o t}(X ; b, y)$ of paths associated to other points $y$ should 
be determined. The idea is that if a suitable classifying space $D^{\text {mot }}$ for such torsors were to be constructed, the Albanese map would merely associate to a point $y$, the class

$$
\left[P^{m o t}(y)\right] \in D^{m o t} .
$$

Coming back to the concrete consideration of realizations, the analytic part of the machinery we need comes from the De Rham fundamental group of $X_{v}:=X \otimes F_{v}$ that depends only on the local arithmetic geometry. Here, $v$ is an archimedean valuation of $F$ not contained in $S$. The definition of this fundamental group requires the use of the category $\operatorname{Un}\left(X_{v}\right)$ of unipotent vector bundles with connections on $X_{v}$. Given any base point $b \in \mathcal{X}\left(R_{v}\right)$, we get a fiber functor $e_{b}: \operatorname{Un}(X) \rightarrow \operatorname{Vect}_{F_{v}}$ to the category of vector spaces over $F_{v}$, and

$$
U^{D R}:=\pi_{1, D R}\left(X_{v}\right):=\operatorname{Aut}^{\otimes}\left(e_{b}\right)
$$

in a rather obvious sense as functors on affine $F_{v}$-schemes. The functor $\pi_{1, D R}\left(X_{v}\right)$ ends up being representable by a pro-unipotent pro-algebraic group. Natural quotients

$$
U_{n}^{D R}:=Z^{n} \backslash U^{D R}
$$

via the descending central series of $U^{D R}$ correspond to restricting $e_{b}$ to the subcategory generated by bundles having index of unipotency $\leq n$. These quotients are unipotent algebraic groups over $F_{v} . U^{D R}$ is endowed with a decreasing Hodge filtration by subgroups:

$$
U^{D R} \supset \cdots \supset F^{n} U^{D R} \supset F^{n+1} U^{D R} \supset \cdots \supset F^{0} U^{D R}
$$

coming from a filtration of the coordinate ring by ideals. There is a comparison isomorphism

$$
U^{D R} \simeq U^{c r} \otimes_{K} F_{v}=\pi_{1, c r}\left(Y_{v}, \bar{b}\right) \otimes_{K} F_{v}
$$

(where $K$ is the maximal absolutely unramified subfield of $F_{v}$ ) with the crystalline fundamental group of the special fiber, defined using unipotent overconvergent iso-crystals. The utility of this is that the Frobenius of the special fiber comes to act naturally on the De Rham fundamental group. Of crucial importance for us is the consideration of De Rham 'path spaces':

$$
P^{D R}(x):=\pi_{1, D R}\left(X_{v} ; x, b\right):=\operatorname{Isom}^{\otimes}\left(e_{b}, e_{x}\right)
$$

consisting of isomorphisms from the fiber functor $e_{b}$ to $e_{x}, x \in X_{v}\left(R_{v}\right)$. These are pro-algebraic varieties also endowed with Hodge filtrations and crystalline Frobenii that are compatible with their structure as right torsors for $U^{D R}$, and 
the point is to study how the data vary with $x$. In fact, they turn out to be classified by a natural 'period space'

$$
U^{D R} / F^{0} U^{D R}
$$

giving us a higher De Rham unipotent Albanese map

$$
j^{D R}: \mathcal{X}\left(R_{v}\right) \rightarrow U^{D R} / F^{0} U^{D R}
$$

that maps $x$ to the class of $P^{D R}(x)$. By passing to (finite-dimensional) quotients $U_{n}^{D R}$, we also have finite-level versions

$$
j_{n}^{D R}: \mathcal{X}\left(R_{v}\right) \rightarrow U_{n}^{D R} / F^{0} U_{n}^{D R}
$$

that fit into a compatible tower

$$
\begin{array}{ccc}
\| & \downarrow \\
\mathcal{X}\left(R_{v}\right) & \rightarrow & U_{n+1}^{D R} / F^{0} \\
\| & & \downarrow \\
\mathcal{X}\left(R_{v}\right) & \rightarrow & U_{n}^{D R} / F^{0} \\
\| & & \downarrow \\
\vdots & \vdots & \vdots \\
\| & & \downarrow \\
\mathcal{X}\left(R_{v}\right) & \rightarrow & U_{2}^{D R} / F^{0}
\end{array}
$$

At the very bottom, $U_{2}^{D R}=H_{1}^{D R}\left(X_{v}\right):=\left(H_{D R}^{1}\left(X_{v}\right)\right)^{*}$ and $j_{2}^{D R}$ is nothing but the logarithm of the usual Albanese map with respect to the base point $b$.

A comparison with the situation over $\mathbb{C}$ easily yields the following:

Theorem 1. For each $n \geq 2$, the image of $j_{n}^{D R}$ is Zariski dense.

This statement can be interpreted as linear independence for multiple polylogarithms of higher genus. It has been pointed out by a referee that this theorem is also proved by Faltings in a preprint [11]. In fact, both Faltings and Akio Tamagawa had indicated to the author earlier the possibility of proving the denseness using trascendental methods in the genus zero case.

Unfortunately, this simple theorem is the only concrete result to be reported on in this paper, the remaining parts being an extended commentary on what else might be expected when allowed considerable optimism. Nevertheless, we proceed to summarize here our observations.

The construction described thus far will have to be compared with one involving pro-unipotent étale fundamental groups that are associated to the 
situation in both local and global settings. That is, after a choice $\bar{F}_{v}$ of an algebraic closure for $F_{v}$, we can associate to the base-change $\bar{X}_{v}:=X_{v} \otimes \bar{F}_{v}$ the pro-unipotent étale fundamental group

$$
U^{e t}:=\pi_{1, \text { ét }}\left(\bar{X}_{v}, b\right)
$$

that classifies unipotent lisse $\mathbb{Q}_{p}$-sheaves on $\bar{X}_{v}$. Furthermore, to each $x$, we can also associate the space of unipotent étale paths $P^{e t}=\pi_{1, \text { ét }}\left(\bar{X}_{v} ; x, b\right)$ which is a torsor for $U^{e t}$. Both carry actions of $G_{v}:=\operatorname{Gal}\left(\bar{F}_{v} / F_{v}\right)$ and the torsor structure is compatible with the action. The torsors that are associated in this way to integral points $x \in \mathcal{X}\left(R_{v}\right)$ have the additional property of being trivialized over a ring $B_{c r}$ of $p$-adic periods (via a non-abelian comparison isomorphism [30], [27]). We classify these torsors using a restricted Galois cohomology set $H_{f}^{1}\left(G_{v}, U^{e t}\right)$. A classifying map defined exactly analogously to the De Rham setting provides a local étale Albanese map

$$
j_{l o c}^{e t}: \mathcal{X}\left(R_{v}\right) \rightarrow H_{f}^{1}\left(G_{v}, U^{e t}\right)
$$

as well as finite-level versions

$$
\left(j_{\text {loc }}^{e t}\right)_{n}: \mathcal{X}\left(R_{v}\right) \rightarrow H_{f}^{1}\left(G_{v}, U_{n}^{e t}\right) .
$$

The connection to $j^{D R}$ goes through a non-abelian extension of Fontaine's Dieudonné functor, interpreted as a morphism of varieties:

$$
D: H_{f}^{1}\left(G_{v}, U_{n}^{e t}\right) \rightarrow U_{n}^{D R} / F^{0}
$$

that fits into a commutative diagram

$$
\begin{aligned}
\mathcal{X}\left(R_{v}\right) & \rightarrow U_{n}^{D R} / F^{0} \\
\searrow & \uparrow \\
& H_{f}^{1}\left(G_{v}, U_{n}^{e t}\right) .
\end{aligned}
$$

The study of global points comes into the picture when we take the basepoint $b$ itself from $\mathcal{X}(R)$, the set of global integral points, and consider the global $\mathbb{Q}_{p}$ pro-unipotent étale fundamental group $\pi_{1, \text { ét }}(\bar{X}, b)$ with the action of $\Gamma=$ $\operatorname{Gal}(\bar{F} / F)$. A choice of an embedding $\bar{F} \hookrightarrow \bar{F}_{v}$ determines an inclusion $G_{v} \hookrightarrow \Gamma$. We have an isomorphism of pro-algebraic groups $\pi_{1 \text {,ét }}(\bar{X}, b) \simeq \pi_{1 \text {,ét }}\left(\bar{X}_{v}, b\right)$ that is compatible with the action of $G_{V}$, so we will allow ourself a minimal abuse of notation and denote the global fundamental group also by $U^{e t}$. Any other global point $y$ then determines a torsor which is denoted $P^{e t}(y)$ (again introducing a bit of confusion with the local object). We denote by $T$ the set of primes 
consisting of $S$ together with all primes dividing the residue characteristic of $v$. The action of $G$ on $U^{e t}$ factors through $G_{T}$, the Galois group of the maximal subfield of $\bar{F}$ unramified over the primes in $T$. Of crucial importance for our purposes is the non-abelian cohomology set

$$
H^{1}\left(G_{T}, U^{e t}\right)
$$

and a natural subset

$$
H_{f}^{1}\left(G_{T}, U^{e t}\right) \subset H^{1}\left(G_{T}, U^{e t}\right)
$$

defined by 'Selmer conditions' at the primes in $T$, which need not be too precise at the primes not equal to $v$, but at $v$, requires the classes to map to $H_{f}^{1}\left(G_{v}, U^{e t}\right)$ under localization. All the cohomology sets thus far discussed can be interpreted as the points of a pro-algebraic variety (over $\left.\mathbb{Q}_{p}\right)$, and $H_{f}^{1}\left(G_{T}, U^{e t}\right)$ is the Selmer variety occurring in the title of this paper. The finite-level versions $H_{f}^{1}\left(G_{T}, U_{n}^{e t}\right)$ and $H_{f}^{1}\left(G_{v}, U_{n}^{e t}\right)$ are algebraic varieties. In this interpretation, the restriction map and the Dieudonné functor become algebraic maps of $\mathbb{Q}_{p^{-}}$ schemes, the target of the latter being the Weil restriction $\operatorname{Res}_{\mathbb{Q}_{p}}^{F_{v}}\left(U^{D R} / F^{0}\right)$, which of course has the property that

$$
\operatorname{Res}_{\mathbb{Q}_{p}}^{F_{v}}\left(U^{D R} / F^{0}\right)\left(\mathbb{Q}_{p}\right)=\left(U^{D R} / F^{0}\right)\left(F_{v}\right) .
$$

We have thus described the fundamental diagram involving the various points of the varieties:

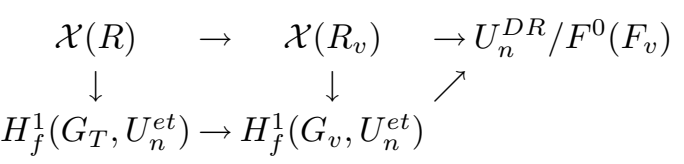

that provides for us the link between Diophantine geometry and the theory of fundamental groups. The reader familiar with the method of Chabauty [4] will recognize here a non-abelian lift of the diagram

$$
\begin{array}{cc}
\mathcal{X}(R) & \rightarrow \quad \mathcal{X}\left(R_{v}\right) \quad \rightarrow \operatorname{Lie}(J) \otimes F_{v} \\
\downarrow & \quad \downarrow \\
J(R) \otimes \mathbb{Q}_{p} & \rightarrow J\left(R_{v}\right) \otimes \mathbb{Q}_{p}
\end{array}
$$

where $J$ is the Jacobian of $\mathcal{X}$, which is essentially the case $n=2$, that is, other than the replacement of the Mordell-Weil group by the Selmer group. The point is that the image of $\mathcal{X}(R)$ inside $U_{n}^{D R} / F^{0}$ thus ends up being contained inside the image of $H_{f}^{1}\left(G_{T}, U_{n}^{e t}\right)$. The desired relation to Diophantine finiteness is expressed by the 
Conjecture 1. Let $F=\mathbb{Q}$ and $X$ be hyperbolic. Then

$$
\operatorname{dim}\left(H_{f}^{1}\left(G_{T}, U_{n}^{e t}\right)\right)<\operatorname{dim}\left(U_{n}^{D R} / F^{0}\right)
$$

for some $n$ (possibly very large).

Whenever one can find an $n$ for which this inequality of dimensions is verified, finiteness of $\mathcal{X}(R)$ follows, exactly as in Chabauty's argument. The only difference is that the analytic functions that occur in the proof are $p$-adic iterated integrals [14] rather than abelian integrals [7].

Unfortunately, this conjecture can be proved at present only when the genus of $X$ is zero [23] and some special genus one situations (with a slight modification of the Selmer variety). However, there is perhaps some interest in the tight connection we establish between the desired statement and various conjectures of 'Birch and Swinnerton-Dyer type.' For example, our conjecture is implied by any one of:

(1) a certain fragment of the Bloch-Kato conjecture [3];

(2) the Fontaine-Mazur conjecture [13];

(3) Jannsen's conjecture on the vanishing of Galois cohomology (when $X$ is affine) $[20]$.

In the case of CM elliptic curves (minus a point) it is easily seen to follow also from the pseudo-nullity of a natural Iwasawa module. All of these implications are rather easy once the formalism is properly set up.

It is amusing to note that these conjectures belong to what one might call 'the structure theory of mixed motives.' That is to say, the usual Diophantine connection for them occurs through the theory of L-functions. It is thus rather surprising that a non-linear (and non-trivial) phenomenon like Faltings' theorem can be linked to their validity. In the manner of physicists, it is perhaps not out of place to view these implications as positive evidence for the structure theory in question.

In relation to the 'anabelian' philosophy, we will explain in the last section how these implications can be viewed as a working substitute for the desired implication 'section conjecture $\Rightarrow$ Faltings' theorem.'

\section{$\S 1$. The De Rham Unipotent Albanese Map}

To start out, we let $L$ be any field of characteristic zero and $S$ be a scheme over $L$. Let $f: X \rightarrow S$ be a smooth scheme over $S$. We denote by $\operatorname{Un}_{n}(X)$ 
the category of unipotent vector bundles with flat connection having index of unipotency $\leq n$. That is, the objects are $\left(\mathcal{V}, \nabla_{\mathcal{V}}\right)$, vector bundles $\mathcal{V}$ equipped with flat connections

$$
\nabla_{\mathcal{V}}: \mathcal{V} \rightarrow \Omega_{X / S} \otimes \mathcal{V}
$$

that admit a filtration

$$
\mathcal{V}=\mathcal{V}_{n} \supset \mathcal{V}_{n-1} \supset \cdots \supset \mathcal{V}_{1} \supset \mathcal{V}_{0}=0
$$

by sub-bundles stabilized by the connection, such that

$$
\left(\mathcal{V}_{i+1} / \mathcal{V}_{i}, \nabla\right) \simeq f^{*}\left(W_{i}, \nabla_{i}\right),
$$

for some bundles with connection $\left(W_{i}, \nabla_{i}\right)$ on $S$. The morphisms are maps of sheaves preserving the connection. Obviously, $\operatorname{Un}_{n}(X)$ is included in $\operatorname{Un}_{m}(X)$ as a full subcategory if $m \geq n$, and we denote by $\operatorname{Un}(X)$ the corresponding union. Let $b \in X(S)$ be a rational point. It determines a fiber functor $e_{b}: \operatorname{Un}(X) \rightarrow \operatorname{Vect}_{S}$ to the category of vector bundles on $S$. Un $(X)$ forms a Tannakian category, and we denote by $<\operatorname{Un}_{n}(X)>$ the Tannakian subcategory of $\operatorname{Un}(X)$ generated by $\operatorname{Un}_{n}(X)$.

Now let $X$ be defined over $L$. Given any $L$-scheme $S$ we follow the standard notation of $X_{S}$ for the base-change of $X$ to $S$. The points $b$ and $x$ in $X(L)$ then determine fiber functors $e_{b}(S), e_{x}(S): \mathrm{Un}\left(X_{S}\right) \rightarrow \operatorname{Vect}_{S}$. We will use the notation $e_{b}^{n}(S)$, etc. to denote the restriction of the fiber functors to $\operatorname{Un}_{n}\left(X_{S}\right)$. The notation $<e_{b}^{n}(S)>$ will be used to denote the restriction to the category $<\operatorname{Un}_{n}(X)>$, The De Rham fundamental group $[10] \pi_{1, D R}(X, b)$ is the prounipotent pro-algebraic group over $L$ that represents the functor on $L$-schemes $S$ :

$$
S \mapsto \operatorname{Aut}^{\otimes}\left(e_{b}(S)\right)
$$

and the path space $\pi_{1, D R}(X ; x, b)$ represents

$$
S \mapsto \operatorname{Isom}^{\otimes}\left(e_{b}(S), e_{x}(S)\right)
$$

We recall that the isomorphisms in the definition are required to respect the tensor-product structure, i.e., if $g \in \operatorname{Isom}\left(e_{b}(S), e_{x}(S)\right)$ and $V, W$ are objects in $\mathrm{Un}\left(X_{S}\right)$, then $g(v \otimes w)=g(v) \otimes g(w)$ for $v \in V_{b}, w \in W_{b}$. It will be convenient to consider also the $L$-module $\operatorname{Hom}\left(e_{b}, e_{x}\right)$ and the $L$-algebra $\operatorname{End}\left(e_{b}\right)$.

We will fix a base point $b$ and denote the fundamental group by $U^{D R}$ and the path space by $P^{D R}(x)$. All these constructions are compatible with base-change. Given three points $x, y, z \in X$, there is a 'composition of paths' map

$$
\pi_{1, D R}(X ; z, y) \times \pi_{1, D R}(X ; y, x) \rightarrow \pi_{1, D R}(X ; z, x)
$$


that induces an isomorphism

$$
\pi_{1, D R}(X ; z, y) \simeq \pi_{1, D R}(X ; z, x)
$$

whenever one picks a $L$-point $p \in \pi_{1, D R}(X ; y, x)$ (if it exists). There is an obvious compatibility when one composes three paths in different orders. In particular, $P^{D R}(x)$ naturally has the structure of a right torsor for $U^{D R}$. We denote by $A^{D R}$ and $\mathcal{P}^{D R}(x)$ the coordinate rings of $U^{D R}$ and $P^{D R}(x)$, respectively. As described in [10], section 10, the $P^{D R}(x)$ fit together to form the canonical torsor

$$
P^{D R} \rightarrow X
$$

which is a right torsor for $X \times{ }_{L} U^{D R}$ and has the property that the fiber over a point $x \in X(L)$ is exactly the previous $P^{D R}(x)$. We denote by $\mathcal{P}^{D R}$ the sheaf of algebras over $X$ corresponding to the coordinate ring of $P^{D R}$. In order to describe this coordinate ring, it will be convenient to describe a universal pro-unipotent pro-bundle associated to the canonical torsor.

The equivalence (loc. cit.) from the category of representations of $U^{D R}$ to unipotent connections on $X$ can be described as

$$
V \mapsto \mathcal{V}:=\left(P^{D R} \times V\right) / U^{D R}
$$

Since $P^{D R}(b)$ is canonically isomorphic to $U^{D R}, \mathcal{V}_{b}$ is canonically isomorphic to $V$. Now let $E$ be the universal enveloping algebra of $\operatorname{Lie} U^{D R}$. Then $E$ has the structure of a co-commutative Hopf algebra and $U^{D R}$ is realized as the grouplike elements in $E$. That is, if $\Delta: E \rightarrow E \hat{\otimes} E$ denotes the co-multiplication of $E$, then $U^{D R}$ is canonically isomorphic to $g \in E$ such that $\Delta(g)=g \otimes g$ (of course, as we vary over points in $L$-algebras). Let $U^{D R}$ act on $E$ on the left, turning $E$ into a pro-representation of $U^{D R}$ : if $I \subset E$ denotes the kernel of the co-unit of $E$, then $E$ is considered as the projective system of the finite-dimensional representations $E[n]:=E / I^{n}$. Define the universal pro-unipotent pro-bundle with connection on $X$ as

$$
\mathcal{E}:=\left(P^{D R} \times E\right) / U^{D R}
$$

which is thus given by the projective system

$$
\mathcal{E}[n]:=\left(P^{D R} \times E[n]\right) / U^{D R} .
$$

Then $\mathcal{E}$ is characterized by the following universal property, which says that it pro-represents the fiber functor: 
If $\mathcal{V}$ is a unipotent vector bundle with connection and $v \in \mathcal{V}_{b}$, then there exists a unique map

$$
\phi_{v}: \mathcal{E} \rightarrow \mathcal{V}
$$

such that $1 \in \mathcal{E}_{b} \rightarrow v \in \mathcal{V}_{b}$.

To see this note that $\mathcal{V}$ is associated to a representation $V$ and that a map $\mathcal{E} \rightarrow \mathcal{V}$ is determined by a map $E=\mathcal{E}_{b} \rightarrow \mathcal{V}_{b} \rightarrow V$ of representations. But then, if $1 \mapsto v$, then $f \in E$ must map to $f v \in V$, so that the map is completely determined by the image of 1 . To reformulate, there is a natural isomorphism

$$
\operatorname{Hom}(\mathcal{E}, \mathcal{V}) \simeq \mathcal{V}_{b}
$$

By the definition of group-like elements, the co-multiplication $\Delta$ is a map of $U^{D R}$ representations. Therefore, there is a map of connections

$$
\Delta: \mathcal{E} \rightarrow \mathcal{E} \otimes \mathcal{E}
$$

which turns $\mathcal{E}$ into a sheaf of co-commutative co-algebras. This map can be also characterized as the unique map $\mathcal{E} \rightarrow \mathcal{E} \otimes \mathcal{E}$ that takes $1 \in \mathcal{E}_{b}$ to $1 \otimes 1 \in(\mathcal{E} \otimes \mathcal{E})_{b}$. We note that there is a map

$$
\mathcal{E}_{x} \rightarrow \operatorname{Hom}\left(e_{b}, e_{x}\right)
$$

defined as follows. Suppose $\mathcal{V}$ is a unipotent vector bundle with connection, $v \in \mathcal{V}_{b}$ and $f \in \mathcal{E}_{x}$. Then $f \cdot v:=\left(\phi_{v}\right)_{x}(f) \in V_{x}$. It is straightforward to check that this map is linear in $v$ and functorial in $\mathcal{V}$. One also checks that all functorial homomorphisms $h: \mathcal{V}_{b} \rightarrow \mathcal{V}_{x}$ arise in this way: Given such an $h$, consider its value $f \in \mathcal{E}_{x}$ on $1 \in \mathcal{E}_{b}$. (There is an obvious way to evaluate $h$ on a pro-unipotent vector bundle with connection. Here as in other places, we are being somewhat sloppy with this passage.) Now given any other $v \in \mathcal{V}$, we have $\phi_{v}: \mathcal{E} \rightarrow \mathcal{V}$ described above. By functoriality, we must have a commutative diagram

$$
\begin{gathered}
\mathcal{E}_{b} \stackrel{h}{\rightarrow} \mathcal{E}_{x} \\
\phi_{v} \downarrow \stackrel{\downarrow}{ } \phi_{v} \\
\mathcal{V}_{b} \stackrel{h}{\rightarrow} \mathcal{V}_{x}
\end{gathered}
$$

Hence, we have

$$
h(v)=h\left(\phi_{v}(1)\right)=\phi_{v}(h(1))=\phi_{v}(f)=f \cdot v,
$$

proving that the value of $h$ is merely the action of $f$. Exactly the same argument with $e_{b}^{n}, e_{x}^{n}$ in place of $e_{b}, e_{x}$ shows that $\mathcal{E}[n]_{x}$ is functorially isomorphic to $\operatorname{Hom}\left(e_{b}^{n}, e_{x}^{n}\right)$. 
Now define

$$
\mathcal{P}:=\mathcal{E}^{*}
$$

which therefore is an ind-unipotent vector bundle with connection. The comultiplication on $\mathcal{E}$ dualizes to endow $\mathcal{P}$ with the structure of a sheaf of commutative algebras over $X$. Since $P^{D R}(x)$ consists of the tensor compatible elements in $\operatorname{Hom}\left(e_{b}, e_{x}\right)=\mathcal{E}_{x}$, we see that this corresponds to the group-like elements in $\mathcal{E}_{x}$ with respect to the co-multiplication, and hence, the algebra homomorphisms $\mathcal{P}(x) \rightarrow L$. Although we've carried out this argument pointwise, it applies uniformly to the whole family as follows: An argument identical to the pointwise one gives us maps

$$
\mathcal{E} \rightarrow \underline{\operatorname{Hom}}\left(\mathcal{V}_{b} \otimes \mathcal{O}_{X}, \mathcal{V}\right)
$$

of sheaves, that together induce an isomorphism of sheaves

$$
\mathcal{E} \simeq \underline{\operatorname{Hom}}\left(e_{b} \otimes \mathcal{O}_{X}, I d\right)
$$

By definition, $P^{D R}$ represents

$$
\underline{\mathrm{Isom}}^{\otimes}\left(e_{b} \otimes \mathcal{O}_{X}, I d\right)
$$

We conclude therefore that $P^{D R}=\operatorname{Spec}_{X}(\mathcal{P})$ and $\mathcal{P}=\mathcal{P}^{D R}$.

Given any pro-algebraic group $G$, we denote by $Z^{n} G$ its descending central series normalized by the indexing $Z^{1} G=G, Z^{n+1} G=\left[G, Z^{n} G\right]$. Corresponding to this, we have the quotient groups, $G_{n}:=G / Z^{n} G$. When the reference to the group is clear from the context, we will often omit it from the notation and write $Z^{n}$ for $Z^{n} G$. A similar convention will apply to the various other filtrations occurring the paper. Another convenient convention we may as well mention here is that we will often omit the connection from the notation when referring to a bundle with connection. That is, $(\mathcal{V}, \nabla \mathcal{V})$ will often be denoted simply by $\mathcal{V}$.

In the case of $U^{D R}$, we define $P_{n}^{D R}(x)$ to be the $U_{n}^{D R}$ torsor obtained by push-out

$$
P_{n}^{D R}(x)=\left(P^{D R}(x) \times U_{n}^{D R}\right) / U^{D R}
$$

Of course it turns out that $P_{n}^{D R}(x)$ represents the functor $\operatorname{Isom}^{\otimes}\left(<e_{b}^{n}>,<\right.$ $\left.e_{x}^{n}>\right)$. The pushout construction can be applied uniformly to the canonical torsor $P^{D R}$ to get $P_{n}^{D R}$ which is a $U_{n}^{D R}$-torsor over $X$.

If we define $\mathcal{P}^{D R}[n]:=\mathcal{E}[n]^{*}$, it gives a filtration

$$
\mathcal{P}^{D R}[0]=\mathcal{O}_{X} \subset \mathcal{P}^{D R}[1] \subset \mathcal{P}^{D R}[2] \subset \cdots
$$


of $\mathcal{P}^{D R}$ by finite-rank sub-bundles that we refer to as the Eilenberg-Maclane filtration. Observe that the multiplication

$$
\mathcal{E} \otimes E \rightarrow \mathcal{E}
$$

is defined by taking $f \in E$ and associating to it the unique homomorphism $\phi_{f}: \mathcal{E} \rightarrow \mathcal{E}$. In particular, when we compose with the projection to $\mathcal{E}[n]$, it factors through to a map

$$
\mathcal{E}[n] \otimes E[n] \rightarrow \mathcal{E}[n] .
$$

Hence, the torsor map

$$
\mathcal{P}^{D R} \rightarrow \mathcal{P}^{D R} \otimes A^{D R}
$$

carries $\mathcal{P}^{D R}[n]$ to $\mathcal{P}^{D R}[n] \otimes A^{D R}[n]$. Therefore, if $P^{D R}$ is trivialized by a point $p \in P^{D R}(S)$ in some $X$-scheme $S$, then the induced isomorphism

$$
i_{p}: \mathcal{P}^{D R} \otimes \mathcal{O}_{S} \simeq A^{D R} \otimes \mathcal{O}_{S}
$$

is compatible with the Eilenberg-Maclane filtration. We should also note that if we embed $L$ into $\mathbb{C}$ and view the whole situation over the complex numbers, then the Eilenberg-Maclane filtration is the one induced by the length of iterated integrals ([19], formula (1.14); this is one version of Chen's $\pi_{1}$ De Rham theorem). In particular, for any complex point $x \in X(\mathbb{C})$, the map obtained by base-change $\mathcal{P}^{D R}[n]_{x} \rightarrow \mathcal{P}_{x}^{D R}$ is injective. Thus, the inclusion $\mathcal{P}^{D R}[n]_{x} \rightarrow \mathcal{P}_{x}^{D R}$ is universally injective. (Proof: Given an $X$-scheme $f: Z \rightarrow X$, any point $z \in Z$ lies over $f(z) \in X$. So injectivity of

$$
f^{*} \mathcal{P}^{D R}[n]_{z} \rightarrow f^{*} \mathcal{P}_{z}^{D R}
$$

reduces to that of

$$
\mathcal{P}^{D R}[n]_{f(z)} \rightarrow \mathcal{P}_{f(z)}^{D R},
$$

which then can be handled through a complex embedding.) Therefore, by the constancy of dimension, we see that the trivializing map $i_{p}$ just mentioned must be strictly compatible with the Eilenberg-Maclane filtration.

Now we let $L=F_{v}$, the completion of a number field $F$ at a nonarchimedean place $v$ and $R_{v}$ the ring of integers in $F_{v}$. Let $X_{v}$ be a smooth curve over $F_{v}$. We will assume that we have a diagram

$$
\begin{aligned}
& X_{v} \hookrightarrow \mathcal{X}_{v} \quad \hookrightarrow \quad \mathcal{X}_{v}^{\prime} \\
& \downarrow \quad \downarrow \quad \downarrow \\
& \operatorname{Spec}\left(F_{v}\right) \hookrightarrow \operatorname{Spec}\left(R_{v}\right)=\operatorname{Spec}\left(R_{v}\right)
\end{aligned}
$$


where $\mathcal{X}_{v}^{\prime}$ is a smooth proper curve over $R_{v}$ and $\mathcal{X}_{v}$ is the complement in $\mathcal{X}_{v}^{\prime}$ of a smooth divisor $\mathcal{D}_{v}$ and $X_{v}$ is the generic fiber of $\mathcal{X}_{v}$. We also denote by $X_{v}^{\prime}\left(\right.$ resp. $\left.D_{v}\right)$ the generic fiber of $\mathcal{X}_{v}^{\prime}\left(\right.$ resp. $\left.\mathcal{D}_{v}\right)$. Letting $k$ denote the residue field of $F_{v}$, we get varieties $Y$ and $Y^{\prime}$ over $k$ as the special fibers of $\mathcal{X}_{v}$ and $\mathcal{X}_{v}^{\prime}$, respectively. Given points $b, x \in \mathcal{X}_{v}\left(R_{v}\right)$, we let $\bar{b}$ and $\bar{x}$ be their reduction to $k$-points of $Y$. Associated to $Y$ and a point $c \in Y(k)$, we have the crystalline fundamental group $\pi_{1, c r}(Y, c)[6]$ which is a pro-unipotent pro-algebraic group over $K$, the field of fraction of the ring of Witt vectors $W$ of $k$. The definition of this group uses the category $\operatorname{Un}(Y)$ of over-convergent unipotent isocrystals [1] on $Y$ which, upon base-change to $F_{v}$, can be interpreted as vector bundles $(\mathcal{V}, \nabla)$ with connections on $X_{v}$ satisfying the unipotence condition of the first paragraph, a convergence condition on each residue disk $] c[$ of point $c \in Y$, and an over-convergence condition near the points of $D_{v}$. For each point $c \in Y(k)$, we get the fiber functor $e_{c}: \mathrm{Un}(Y) \rightarrow \operatorname{Vect}_{K}$ which, again upon base-change to $F_{v}$, associates to a pair $(\mathcal{V}, \nabla)$ the set of flat sections $\mathcal{V}(] c[)^{\nabla=0}$ on the residue disk $] c\left[\left([2]\right.\right.$, p. 26). $\pi_{1, c r}(Y, c)$ then represents the group of tensor automorphisms this fiber functor. Similarly, using two points and the functor $\operatorname{Isom}^{\otimes}\left(e_{c}, e_{y}\right)$, we get the $\pi_{1, c r}(Y, c)$-torsor $\pi_{1, c r}(Y ; y, c)$ of crystalline paths from $c$ to $y$. As before, we will fix a $c$ in the discussion and use the notation $U^{c r}=\pi_{1, c r}(Y, c), P^{c r}(y)=\pi_{1, c r}(Y ; y, c)$. In fact, if we let $c=\bar{b}$ and $y=\bar{x}$, then we have canonical isomorphisms ([6], prop. 2.4.1)

$$
\begin{aligned}
U^{D R} & \simeq U^{c r} \otimes_{K} F_{v} \\
P^{D R}(x) & \simeq P^{c r}(\bar{x}) \otimes_{K} F_{v}
\end{aligned}
$$

which are compatible with the torsor structures and, more generally, composition of paths. The key point is that all algebraic unipotent bundles satisfy the (over-)convergence condition. Because $P^{c r}(y)$ is functorial, it carries a pro-algebraic automorphism

$$
\phi: P^{c r}(y) \simeq P^{c r}(y)
$$

induced by the $q=|k|$-power map on $\mathcal{O}_{Y}$. The various Frobenius maps are also compatible with the torsor structures. The comparison isomorphism then endows $P^{D R}$ with a map that we will again denote by $\phi$. Given a point $x \in$ $X_{v}\left(R_{v}\right)$ that lies in the residue disk $] \bar{b}[$, we have

$$
P^{D R}(x) \simeq U^{c r} \otimes_{K} F_{v}
$$

and it is worth chasing through the definition to see precisely what the path is on the left-hand side corresponding to the identity in $U^{c r}$ : Given a unipotent 
bundle $(\mathcal{V}, \nabla)$, we construct an isomorphism $\mathcal{V}_{b} \simeq \mathcal{V}_{x}$ by finding the unique flat section $s$ over $] \bar{b}$ [ with initial value at $b$ equal to $v_{b}$. Then the image of $v_{b}$ under the isomorphism (i.e., the path) is $s(x)$, the value of this flat section at $x$. Since $\phi$ also respects the subcategories $\operatorname{Un}_{n}(Y)$ consisting of the overconvergent isocrystals with index of unipotency $\leq n$, it preserves the Eilenberg-Maclane filtration on the $\mathcal{P}^{D R}(x)$.

According to [32], theorem E, $\mathcal{P}^{D R}$ possesses a Hodge filtration. This is a filtration

$$
\mathcal{P}^{D R}=F^{0} \mathcal{P}^{D R} \supset \cdots \supset F^{n} \mathcal{P}^{D R} \supset F^{n+1} \mathcal{P}^{D R} \supset \cdots
$$

by sub- $\mathcal{O}_{X}$-modules that is compatible with the multiplicative structure. In particular, the $F^{i}$ are ideals. This induces a filtration on the fibers $\mathcal{P}^{D R}(x)$ and in particular on the coordinate ring $A^{D R}$ of $U^{D R}$. The Hodge filtration is compatible with the torsor structure in that if the tensor product $\mathcal{P}^{D R} \otimes_{L} A^{D R}$ is endowed with the tensor product filtration

$$
F^{n}\left(\mathcal{P}^{D R} \otimes_{L} A^{D R}\right)=\Sigma_{i+j=n}\left(F^{i} \mathcal{P}^{D R}\right) \otimes_{L}\left(F^{j} A^{D R}\right)
$$

then the torsor map

$$
\mathcal{P}^{D R} \rightarrow \mathcal{P}^{D R} \otimes_{L} A^{D R}
$$

is compatible with the filtration. As in the case of the Eilenberg-Maclane filtration, if we utilize their descriptions over $\mathbb{C}[19]$, we see immediately that the inclusions $F^{i} \mathcal{P}^{D R_{\hookrightarrow}} \mathcal{P}^{D R}$ are also universally injective, and that if we restrict the Hodge filtration to the terms in the Eilenberg-Maclane filtration, then the rank of $F^{i} \mathcal{P}^{D R}[n]$ is equal to the dimension of $F^{i} A^{D R}[n]$. Since the $F^{i}$ are ideals, there are corresponding sub- $X$-schemes

$$
F^{i} P^{D R} \text {, }
$$

where $F^{-i+1} \mathcal{P}^{D R}$ is the defining ideal for $F^{i} P^{D R}$. In fact, $F^{0} P^{D R}$ is a $F^{0} U^{D R_{-}}$ torsor. To see the action, note that compatibility implies that the torsor map takes $F^{1} \mathcal{P}^{D R}$, the defining ideal for $F^{0} P^{D R}$, to

$$
F^{1} \mathcal{P}^{D R} \otimes A^{D R}+\mathcal{P}^{D R} \otimes F^{1} A^{D R},
$$

the latter being exactly the defining ideal for $F^{0} P^{D R} \times F^{0} U^{D R}$ inside $P^{D R} \times$ $U^{D R}$. Thus, we get the action map

$$
F^{0} P^{D R} \times F^{0} U^{D R} \rightarrow F^{0} P^{D R} .
$$

On the other hand, assume we have a point $p \in F^{0} P^{D R}(S)$ in some $X$-scheme $S$. Then the corresponding isomorphism

$$
i_{p}: \mathcal{P}^{D R} \otimes \mathcal{O}_{S} \simeq A_{D R} \otimes \mathcal{O}_{S}
$$


is compatible with the Hodge filtration and hence, strictly compatible because of the universal injectivity mentioned above and the equality of ranks (of course when restricted to the finite-rank terms of the Eilenberg-Maclane filtration). Therefore, it induces an isomorphism

$$
i_{p}: F^{0} U^{D R} \simeq F^{0} P^{D R},
$$

proving the torsor property. (The remaining compatibilities are obvious from the corresponding properties for $P^{D R}$.) Regarding these trivializations, we note that if a point $p \in P^{D R}(S)$ induces an isomorphism as above compatible with the Hodge filtration, then since $p$ is recovered as $e v_{0} \circ i_{p}$, where $e v_{0}$ : $A_{D R} \otimes \mathcal{O}_{S} \rightarrow \mathcal{O}_{S}$ is the origin of $U_{S}^{D R}$, we see that $p \in F^{0} P^{D R}$.

We will need some abstract definitions corresponding to the situation described. Given an $F_{v}$-scheme $Z$, by a torsor over $Z$ for $U^{D R}$, we mean a right $U_{Z}^{D R}$ torsor $T=\operatorname{Spec}(\mathcal{T})$ over $Z$ endowed with an 'Eilenberg-Maclane' filtration:

$$
\mathcal{T}[0] \subset \mathcal{T}[1] \subset \cdots
$$

and a 'Hodge' filtration

$$
\mathcal{T}=F^{0} \mathcal{T} \supset F^{1} \mathcal{T} \supset \cdots
$$

of the coordinate ring. The Eilenberg-Maclane filtration should consist of locally-free $\mathcal{O}_{Z}$-modules of finite rank equal to $\operatorname{dim}_{F_{v}} A^{D R}[n]$ with the property that $\mathcal{T}[n] \hookrightarrow \mathcal{T}$ is universally injective. Furthermore, each $F^{i} \mathcal{T}[n] \hookrightarrow \mathcal{T}[n]$ must be universally injective and have rank equal to $\operatorname{dim} F^{i} A^{D R}[n]$. Both filtrations are required to be compatible with the torsor structure in slightly different senses, namely, that $\mathcal{T}[n]$ must be carried to $\mathcal{T}[n] \otimes A^{D R}[n]$ while $F^{m} \mathcal{T}[n]$ is to be taken to $\Sigma_{i+j=m} F^{i} \mathcal{T} \otimes F^{j} A^{D R}$. The torsor $T$ must also carry a 'Frobenius' automorphism of $Z$-schemes $\phi: T \rightarrow T$ (we will denote all of them by the same letter) which is required to be compatible with the torsor structure in the sense that $\phi(t) \phi(u)=\phi(t u)$ for points $t$ of $T$ and $u$ of $U$. We require that $\phi$ preserves the Eilenberg-Maclane filtration, although not necessarily the Hodge filtration. We will call the torsor $T$ admissible, if it is separately trivializable for the Frobenius structure and the Hodge filtration. That is, we are requiring that there is a point $p^{c r} \in T(Z)$ which is invariant under the Frobenius, and also that there is a point $p^{D R} \in F^{0} T(Z)$.

The following lemma follows from the argument of [2], cor. 3.2.

Lemma 1. Let $T$ any torsor for $U^{D R}$ over an affine scheme $Z$. Then $T$ is uniquely trivializable with respect to the $\phi$-structure. That is, there is a unique point $p_{T}^{c r} \in T(Z)$ which is invariant under $\phi$. 
The key fact is that the map $U^{D R} \rightarrow U^{D R}, x \mapsto \phi\left(x^{-1}\right) x$ is surjective. Obviously, the canonical path is the identity for the trivial torsor $U_{Z}^{D R}$.

Combining this lemma with the earlier remarks and the unipotence of $F^{0} U^{D R}$, we get that for any affine $X$-scheme $Z, P_{Z}^{D R}$ is an admissible torsor.

We say two admissible torsors are isomorphic if there is a torsor isomorphism between them that is simultaneously compatible with the Frobenius and Hodge filtration.

Let $T$ be an admissible $U^{D R}$ torsor over an $F_{v}$-algebra $L$. Choose a trivialization $p_{T}^{H} \in F^{0} T(L)$. There then exists a unique element $u_{T} \in U^{D R}$ such that $p_{T}^{c r} u_{T}=p_{T}^{H}$. Clearly, a different choice of $p^{H}$ will change the result only by right multiplication with an element of $F^{0} U^{D R}$. Thus, we get an element $\left[u_{T}\right] \in U^{D R} / F^{0} U^{D R}$ which is independent of the choice of $p_{T}^{H}$.

Proposition 1. The map

$$
T \mapsto\left[u_{T}\right]
$$

defines a natural bijection from the isomorphism classes of admissible torsors to $U^{D R} / F^{0} U^{D R}$. That is to say, the scheme $U^{D R} / F^{0} U^{D R}$ represents the functor that assigns to each $F_{v}$ algebra $L$ the isomorphism classes of admissible torsors on $L$.

Proof. The map defined is clearly functorial, so we need only check bijectivity on points. Suppose $\left[u_{T}\right]=\left[u_{S}\right]$. Then there exists a $u^{0} \in F^{0} U^{D R}$ such that $u_{S}=u_{T} u^{0}$. The elements $p_{T}^{c r}$ and $p_{S}^{c r}$ already determine $\phi$-compatible isomorphisms

$$
f: S \simeq U^{D R} \simeq T ; \quad p_{S}^{c r} u \mapsto u \mapsto p_{T}^{c r} u
$$

It suffices to check that this isomorphism is compatible with the Hodge filtration. But we already know that the map

$$
h: p_{S}^{H} g \mapsto g \mapsto p_{T}^{H} g
$$

is compatible with the Hodge filtration, and writing the $\phi$-compatible map $f$ with respect to the $p^{H}$ 's, we get

$$
p_{S}^{H} g=p_{S}^{c r} u_{S} g \mapsto p_{T}^{c r} u_{S} g=p^{H} u_{T}^{-1} u_{S} g=p^{H} u^{0} g .
$$

That is, it corresponds merely to a different choice $p^{H} u^{0}$ of the trivialization. So we need to check that $p^{H} u^{0} \in F^{0} T$, i.e., that it is a Hodge trivialization. Its values on the coordinate ring of $T$ is expressed through the composition

$$
\mathcal{T} \rightarrow \mathcal{T} \otimes A^{D R} \stackrel{p^{H} \times u^{0}}{\rightarrow} L
$$


But then, since the action takes $F^{1} \mathcal{T}$ to

$$
F^{1} \mathcal{T} \otimes A^{D R}+\mathcal{T} \otimes F^{1} A^{D R},
$$

$F^{1} \mathcal{T}$ is clearly killed by this evaluation, i.e., $p^{H} u^{0} \in F^{0} T$.

Thus the map of functors described is injective. To see that it is surjective, note that for any $Z$, we have

$$
\left[U^{D R} / F^{0} U^{D R}\right](Z)=U^{D R}(Z) / F^{0} U^{D R}(Z) .
$$

Now, given $g \in U^{D R}$, we consider $U^{D R}$ with the same Hodge filtration, but with the automorphism twisted to $\phi^{g}(h)=g^{-1} \phi(g h)$. This automorphism is compatible with right multiplication, that is, the right torsor structure on $U^{D R}$ :

$$
\phi^{g}(h k)=g^{-1} \phi(g h k)=g^{-1} \phi(g h) \phi(k)=\phi^{g}(h) \phi(k)
$$

Thus, we end up with an admissible torsor $T_{g}$. The $\phi^{g}$-fixed element here is clearly $g^{-1}$, and thus, $u_{T_{g}}=g$, as desired.

Already we have the language necessary to define the De Rham unipotent Albanese map:

$$
j^{D R} \mathcal{X}_{v}\left(R_{v}\right) \rightarrow U^{D R} / F^{0} U^{D R}
$$

by

$$
j^{D R}(x):=\left[P^{D R}(x)\right] .
$$

The finite-level versions $j_{n}^{D R}$ are defined by composing with the natural projections

$$
U^{D R} / F^{0} U^{D R} \rightarrow U_{n}^{D R} / F^{0} U_{n}^{D R} .
$$

There is a parallel discussion of admissible torsors for $U_{n}^{D R}$ out of which one can extract the interpretation of

$$
j_{n}^{D R}: \mathcal{X}_{v}\left(R_{v}\right) \rightarrow U_{n}^{D R} / F^{0} U_{n}^{D R}
$$

as

$$
x \mapsto\left[P_{n}^{D R}(x)\right]
$$

In order to describe the map, it will be convenient to have a rather explicit construction of $U^{D R}$ and $P^{D R}$. We will carry this out assuming $X_{v}$ is affine, which is the only case we will need. The construction depends on the choice $\alpha_{1}, \alpha_{2}, \cdots, \alpha_{m}$ of global algebraic differential forms representing a basis 
of $H_{D R}^{1}\left(X_{v}\right)$. So here, we have $m=2 g+s-1$, where $s$ is the order of $X_{v}^{\prime} \backslash X_{v}$. Corresponding to this choice, there is a free non-commutative algebra

$$
F_{v}<A_{1}, \ldots, A_{m}>
$$

generated by the symbols $A_{1}, A_{2}, \ldots, A_{m}$. Thus, it is the group algebra of the free group on $m$ generators. Let $I$ be its augmentation ideal. The algebra $F_{v}<A_{1}, \ldots, A_{m}>$ has a natural comultiplication map $\Delta$ with values $\Delta\left(A_{i}\right)=$ $A_{i} \otimes 1+1 \otimes A_{i}$. Now we consider the completion

$$
E:=\lim _{\longleftarrow} F_{v}<A_{1}, \ldots, A_{m}>/ I^{n}
$$

$\Delta$ extends naturally to a comultiplication $E \rightarrow E \hat{\otimes} E$. We can also consider the quotients

$$
E[n]:=E / I^{n+1}=F_{v}<A_{1}, \ldots, A_{m}>/ I^{n+1}
$$

that carry induced maps

$$
E[n] \rightarrow \oplus_{i+j=n} E[i] \otimes E[j]
$$

Now let $\mathcal{E}$ be the pro-unipotent pro-vector bundle $E \otimes \mathcal{O}_{X_{v}}$ with the connection $\nabla$ determined by

$$
\nabla f=-\Sigma_{i} A_{i} f \alpha_{i}
$$

for constant sections $f \in E$. We also have the finite-level quotients $\mathcal{E}[n]:=$ $E[n] \otimes \mathcal{O}_{X_{v}}$. This construction ends up as the 'universal' pro-unipotent probundle with connection in the sense of the beginning paragraphs, justifying the conflation of notation. To see this, we need a lemma.

Lemma 2. Let $(V, \nabla)$ be a unipotent bundle with flat connection on $X_{v}$ of rank $r$. Then there exist strictly upper-triangular matrices $N_{i}$ such that

$$
(V, \nabla) \simeq\left(\mathcal{O}_{X}^{r}, d+\Sigma_{i} \alpha_{i} N_{i}\right)
$$

Proof of lemma. Since $X_{v}$ is affine and $(V, \nabla)$ is unipotent, by choosing vector bundle splittings of the filtration, there exists a trivialization $V \simeq \mathcal{O}_{X}^{r}$ such that $\nabla$ takes the form $d+\omega$ for some strictly upper-triangular $n \times n$ matrix of 1-forms $\omega$. It will be convenient to write $\omega=\Sigma \omega_{i j} E_{i j}$ where $E_{i j}$ is the elementary $n \times n$ matrix with a 1 in the $(i, j)$-entry and zero elsewhere, and $\omega_{i j}=0$ unless $j>i$. Recall that a gauge transformation $G$ will change the connection matrix by

$$
\omega \mapsto G^{-1} \omega G+G^{-1} d G
$$


For a gauge transformation of the form $G=I-a E_{i j}(j \neq i)$, we have $G^{-1}=I+a E_{i j}$ while $G^{-1} d G=d a E_{i j}$. We wish to perform a series of gauge transformations so that each entry $\omega_{i j}$ is replaced by a linear combination of the $\alpha_{i}$ 's and $\beta_{j}$ 's. Now, any single one-form can be written as such a combination after adding an exact form. We will show how to change $\omega_{i j}$ by induction on $j-i$. Assume we are done for $j-i<c$. So let $i, j$ satisfy $j-i=c$. First find $a$ such $\omega_{i j}+d a$ is a linear combination of the $\alpha_{i}$ and $\beta_{j}$. Now consider the gauge transformation $G=I-a E_{i j}$. We compute

$$
G^{-1} \omega=\left(I+a E_{i j}\right)\left(\Sigma \omega_{k l} E_{k l}\right)=\omega+\Sigma_{l} \omega_{j l} a E_{i l}=: \omega^{\prime}
$$

Note the only non-zero $l$ occurring in the sum are strictly bigger than $j$. Thus, $\omega_{\mu \nu}^{\prime} \neq \omega_{\mu \nu}$ only occurs for $\mu=i$ and $\nu>j$, that is, for $\nu-\mu>j-i$. On the other side,

$$
\omega^{\prime} G=\left(\Sigma \omega_{k l}^{\prime} E_{k l}\right)\left(I-a E_{i j}\right)=\omega^{\prime}-\Sigma \omega_{k i}^{\prime} E_{k j}
$$

and all the $k$ appearing in the sum are $<i$. Therefore, we see that all the entries $\omega_{k l}^{G}$ of $\omega^{G}:=G^{-1} \omega G$ are equal to $\omega_{k l}$ for $l-k \leq c$. On the other hand, $G^{-1} d G=d a E_{i j}$. Performing such a gauge transformation for each $i, j$ such that $j-i=c$ clearly achieves what we want.

Now we can formulate the universal property of $\mathcal{E}$ :

Lemma 3. Given any object $(V, \nabla)$ in $U n\left(X_{v}\right)$ together with a an element $v \in V_{b}$ (the fiber at b), there exists a unique morphism $\phi: \mathcal{E} \rightarrow V$ in $U n\left(X_{v}\right)$ such that $1 \in \mathcal{E}_{b} \mapsto v$. That is, $\mathcal{E}$ is the same as the bundle with the same notation from the beginning of the section.

Proof. First we will show uniqueness. For this, we can choose an embedding of $F_{v}$ into the complex numbers and assume that everything is defined over $\mathbb{C}$ (and for convenience, we will omit the base-change to $\mathbb{C}$ from the notation). By uniqueness of solutions to differential equations with given initial condition, we see that any map of bundles $\phi: \mathcal{E} \rightarrow V$ is completely determined by its value at $b$. (Actually, for this, we needn't go to $\mathbb{C}$. It suffices to use uniqueness of formal solutions.) So we need to check that the value of the map is determined at $b$ by the given conditions. Let $\pi$ be the topological fundamental group of $X_{v}(\mathbb{C})$ and let $\mathbb{C}[\pi]$ be the complex group algebra of $\pi$. The holonomy transformations give an action of $\mathbb{C}[\pi]$ on the fibers $\mathcal{E}_{b}$ and $V_{b}$. Since $\phi$ respects the connections, $\phi_{b}$ is equivariant for this action. But over $\mathbb{C}$, one can compute the holonomy using iterated integrals. Given a word $w=A_{i_{1}} A_{i_{2}} \cdots A_{i_{n}}$ in the $A_{i}$ 
and a piece-wise smooth path $\gamma$, denote by $\int_{\gamma} \alpha_{w}$, the iterated integral

$$
\int_{\gamma} \alpha_{i_{1}} \alpha_{1_{2}} \cdots \alpha_{i_{n}}
$$

We are using the normalization whereby if $\gamma^{*}\left(\omega_{i}\right)=f_{i}(t) d t$, then

$$
\begin{aligned}
\int_{\gamma} \omega_{1} \omega_{2} \cdots \omega_{n}= & \int_{0}^{1} f_{1}\left(t_{1}\right) \int_{0}^{t_{1}} f_{2}\left(t_{2}\right) \int_{0}^{t_{2}} f_{3}\left(t_{3}\right) \int \cdots \\
& \int_{0}^{t_{n-1}} f_{n}\left(t_{n}\right) d t_{n} d t_{n-1} \cdots d t_{1} .
\end{aligned}
$$

On a contractible open set $U$, if we pick a base point $c$ and define the function $G_{c}(z)$ with values in $E$ on $U$ by

$$
G_{c}(z)=\Sigma_{w}\left(\int_{c}^{z} \alpha_{w}\right)[w],
$$

where the iterated integral occurs along any path from $c$ to $z$, then $d G_{c}=$ $\Sigma_{i} \alpha_{i} A_{i} G_{c}$ so that $G_{c}(z)$ can be viewed as a flat section of $\mathcal{E}$. In particular, for the holonomy around a loop $\gamma$ based at $b$,

$$
\gamma \cdot f=\Sigma_{w}\left(\int_{\gamma} \alpha_{w}\right)[w] f
$$

for $f \in E=\mathcal{E}_{b}$. Thus, we must have

$$
\phi\left[\Sigma_{w}\left(\int_{\gamma} \alpha_{w}\right)[w] 1\right]=\gamma \phi(1)=\gamma v
$$

Now consider the map $\mathbb{C}[\pi] \rightarrow E$ given by the formula

$$
\gamma \mapsto G_{\gamma}:=\Sigma_{w}\left(\int_{\gamma} \alpha_{w}\right)[w]
$$

(We will use the natural notation $\int_{\gamma} \alpha_{w}:=\Sigma_{i} c_{i} \int_{\gamma_{i}} \alpha_{w}$ if $\gamma=\Sigma_{i} c_{i} \gamma_{i}, c_{i} \in$ $\mathbb{C}, \gamma_{i} \in \pi$.) To conclude the proof of uniqueness, we just need to see that the composition to each of the finite-dimensional quotients $E_{n}$ is surjective. But if not, we would have a non-trivial linear relation between the $\int \alpha_{w}$ regarded as functions on $\mathbb{C}[\pi]$. We show by induction on the length of $w$ that they are in fact linearly independent. For this, let $a_{1}, \ldots, a_{m}$ be elements of $\mathbb{C}[\pi]$ whose classes in homology form a dual basis to the $\alpha_{i}$. Assume

$$
\Sigma_{|w| \leq n} c_{w} \int_{\gamma} \alpha_{w}=0
$$


for all $\gamma \in \pi$. Write this as

$$
\Sigma_{|w| \leq n-1}\left(\Sigma_{i} c_{A_{i} w} \int_{\gamma} \alpha_{A_{i} w}\right)=0
$$

and apply it to $a_{j} \gamma$. Then we have

$$
\Sigma_{|w| \leq n-1}\left(\Sigma_{i} c_{A_{i} w} \int_{a_{j} \gamma} \alpha_{i} \alpha_{w}\right)=0
$$

The co-product formula for iterated integrals [5] can then be applied to give

$$
\Sigma_{|w| \leq n-1}\left[\Sigma_{i} c_{A_{i} w}\left(\operatorname{deg}\left(a_{j}\right) \int_{\gamma} \alpha_{i} \alpha_{w}+\int_{a_{j}} \alpha_{i} \int_{\gamma} \alpha_{w}+\cdots\right)\right]=0,
$$

where $\operatorname{deg}\left(\Sigma c_{\gamma}[\gamma]\right)=\Sigma c_{\gamma}$ on elements of $\mathbb{C}[\pi]$. From this, we subtract $\left(\operatorname{deg}\left(a_{j}\right)\right.$ times) the original relation to get

$$
\Sigma_{|w|=n-1} c_{A_{j} w} \int_{\gamma} \alpha_{w}+\Sigma_{|w| \leq n-2} b_{w} \int_{\gamma} \alpha_{w}=0
$$

for some constants $b_{w}$. Since this holds for all $\gamma$, by induction, we have $c_{A_{j} w}=0$ for $|w|=n-1$, and hence $c_{w}=0$ for $|w|=n$ when the argument is applied to each $A_{j}$. Then again by induction, all the $c_{w}$ must vanish.

The existence part is easy. We may assume that $(V, \nabla)$ is of the form $\left(\mathcal{O}_{X_{v}}^{r}, d-\Sigma_{i} \alpha_{i} N_{i}\right)$ as in the previous lemma, except we have changed $N_{i}$ to $-N_{i}$. Now given the vector $v \in V_{b}$, define the map $\mathcal{E} \rightarrow V$ that sends a section $\Sigma f_{w}[w]$ of $\mathcal{E}$ to $\Sigma_{w} f_{w} N_{w} v$ where the notation is that if a word has the form $w=$ $A_{i_{1}} A_{i_{2}} \cdots A_{i_{n}}$, then $N_{w}$ is the matrix $N_{i_{1}} N_{i_{2}} \cdots N_{i_{n}}$. This map is obviously compatible with the connection and takes the constant section 1 to the constant section $v$ (in particular, at the point $b$ ).

As mentioned already, another way of phrasing the lemma is to say that there is a canonical isomorphism:

$$
\operatorname{Hom}(\mathcal{E}, V) \simeq V_{b}
$$

In particular, $\operatorname{Hom}(\mathcal{E}, \mathcal{E}) \simeq \mathcal{E}_{b}=E$ and it is easily checked that this isomorphism is compatible with the algebra structure.

Recall the evaluation fiber functor $e_{b}: \operatorname{Un}\left(X_{v}\right) \rightarrow \operatorname{Vect}_{F_{v}}$. Since $\mathcal{E}$ is the universal pro-unipotent vector bundle with connection, $E=\mathcal{E}_{b}$ is canonically isomorphic to $\operatorname{End}\left(e_{b}\right)$ and $E=\mathcal{E}_{x}$ is canonically isomorphic to $\operatorname{Hom}\left(e_{b}, e_{x}\right)$. 
It is equally easy to see that $E[n]$ canonically isomorphic to $\operatorname{End}\left(e_{b}^{n}\right)$ and $\operatorname{Hom}\left(e_{b}^{n}, e_{x}^{n}\right)$.

If we examine the co-multiplication $\Delta: \mathcal{E} \rightarrow \mathcal{E} \otimes \mathcal{E}$ associated to the $\mathcal{E}$ as a universal object, that is, the unique map that sends 1 to $1 \otimes 1$, by the definition of the connection on $\mathcal{E}$, it takes $A_{i}$ to $A_{i} \otimes 1+1 \otimes A_{i}$. That is, the co-multiplication is compatible with the one defined formally on $E=\mathcal{E}_{b}$. So we also have that $\operatorname{Aut}^{\otimes}\left(e_{b}\right)$ and $\operatorname{Isom}^{\otimes}\left(e_{b}, e_{x}\right)$ are represented by the group-like elements in $\mathcal{E}_{b}=E=\mathcal{E}_{x}$ and that $\mathcal{E}^{*}$, the dual bundle of $\mathcal{E}$, with its structure of a commutative algebra is nothing but $\mathcal{P}^{D R}$.

Now $\mathcal{P}^{D R}$ carries a Hodge filtration by sub-bundles, but its explicit description will be unnecessary for our purposes. All we will need is that $F^{0} P$ is a torsor for the group $F^{0} U^{D R}$, and hence, can be trivialized over $X_{v}$ in the case it is affine.

Proof of theorem 1. First note that if $X_{v}$ happens to be compact, we can delete an $R_{v}$ point $x_{0}$ away from the residue disk of $b$ to get $\mathcal{X}_{v}^{0}=$ $\mathcal{X}_{v} \backslash x_{0}$, still equipped with an $R_{v}$ point $b$. Since restriction embeds the unipotent bundles with flat connection on $X_{v}$ as a full subcategory of the unipotent bundles on $X_{v}^{0}$ (see, for example, [2], cor. 2.15), we get a surjection $\pi_{1, D R}\left(X_{v}^{0}, b\right) \rightarrow \pi_{1, D R}\left(X_{v}, b\right)$. This map is compatible with the Frobenius because the comparison isomorphism is induced by the functor that associates to a unipotent bundle with connection $\mathcal{E}$ the overconvergent isocrystal $j^{\dagger} \mathcal{E}^{r i g}$ ([6], Prop. 2.4.1), which is obviously compatible with maps of varieties.

Functoriality of the Hodge filtration can be deduced either from an examinination of Wojkowiak's construction or the usual functoriality over $\mathbb{C}$ [18]. Of course, an identical argument can be applied to the path spaces, giving us a commutative diagram

$$
\begin{array}{cc}
\mathcal{X}^{0}\left(R_{v}\right) & \rightarrow \pi_{1, D R}\left(X_{v}^{0}, b\right) / F^{0} \\
\downarrow & \downarrow \\
\mathcal{X}\left(R_{v}\right) & \rightarrow \pi_{1, D R}\left(X_{v}, b\right) / F^{0}
\end{array}
$$

where the right vertical map is surjective. Therefore, it suffices to prove the theorem for an affine curve.

In fact, we will show that the image of the residue disk $] \bar{b}$ [is already Zariski dense. Now, as described above, there exists a canonical $U^{D R}$ torsor $P^{D R}$ on $X_{v}$ with the property that the fiber over $x$ is exactly $P^{D R}(x)$ which is, in fact, admissible. This is because of the unique Frobenius invariant element and the fact that $F^{0} P^{D R}$ is a $F^{0} U^{D R}$-torsor, and hence, has a section over an affine scheme. (Since vector groups have no non-trivial torsors over affine schemes, neither do unipotent groups.) Choose such a trivialization $p^{H} \in F^{0} P^{D R}\left(X_{v}\right)$. 
It is important to note here that $F^{0} P^{D R}$ becomes thereby algebraically trivialized. We can compare this with any other algebraic trivialization $g \in P^{D R}\left(X_{v}\right)$. That is, there exists an algebraic map $\gamma: X_{v} \rightarrow U^{D R}$ such that $g \gamma=p^{H}$. For any $x \in X_{v}$, we have a point $u(x) \in U^{D R}$ such that $p^{c r}(x) u(x)=g(x)$ so that $p^{c r}(x) u(x) \gamma(x)=p^{H}(x)$. That is, $j^{D R}(x)=[u(x) \gamma(x)]$. Therefore, it suffice to show that the image of $x \mapsto u(x) \gamma(x)$ is Zariski dense in $U^{D R}(X)$. As previously discussed, given $(V, \nabla)$, on $] \bar{b}\left[\right.$, the element $p^{c r}(x)$ is obtained from the diagram

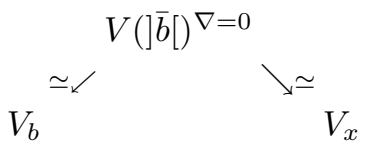

as the inverse of the left arrow followed by the right arrow. We proceed now to describe the various objects in local coordinates to obtain the desired result.

Take $g$ to be the trivialization of $P^{D R}$ determined by the previously described trivialization of $\mathcal{E}$. That is, since we have identified $\operatorname{Isom}^{\otimes}\left(e_{b}, e_{x}\right)=E$, the element in question is $1 \in E$. For $x \in] \bar{b}[u(x)$ is then identified with the value of the function $u:] \bar{b}[\rightarrow E$ that is the unique horizontal section of $\mathcal{E}$ convergent on $] \bar{b}\left[\right.$ such that $u(b)=1$. Write $u=\Sigma_{w} u_{w}[w]$, where $w$ runs over the words in $A_{i}$. We will show:

Lemma 4. The $u_{w}$ are linearly independent over the algebraic functions on $X_{v}$.

It is easy to see that this lemma implies the theorem:

We have

$$
j^{D R}(x)=\Sigma_{w} j_{w}(x)[w]=u(x) \gamma(x)=\Sigma\left[\sigma_{w^{\prime} w^{\prime \prime}=w} u_{w^{\prime}}(x) \gamma_{w^{\prime \prime}}(x)\right][w],
$$

where $\gamma=\Sigma_{w} \gamma_{w}[w]$ If the image of $j_{n}^{D R}$ were in a Zariski closed subspace, we would have a linear relation among the $j_{w}$, by the description we have given of the coordinate ring of $U^{D R}$. Let us see by induction on the length of $w$ that the $j_{w}$ are linearly independent. Assume we have a relation

$$
\Sigma_{|w| \leq n} c_{w} j_{w}=0
$$

Then

$$
\Sigma_{|w| \leq n} c_{w}\left[\Sigma_{w^{\prime} w^{\prime \prime}=w} u_{w^{\prime}} \gamma_{w^{\prime \prime}}\right]=0 .
$$

This would give us a relation of the form

$$
\Sigma_{|w| \leq n} a_{w} u_{w}=0
$$


where the $a_{w}$ are algebraic functions. Hence, all $a_{w}=0$. But for $|w|=n$, we have $a_{w}=c_{w} \gamma_{0}$ and $\gamma_{0}=1$ because $\gamma$ is group-like. Therefore, we must have $c_{w}=0$ for all $|w|=n$. The result follows by induction.

So we need only give the

Proof of lemma. The proof here is entirely similar to the uniqueness part of Lemma 4. Even though the $u_{w}$ are convergent on $] \bar{b}$ [ we can regard them as formal power series and show independence over the algebraic functions in there. For this, we choose an embedding $F_{v} \hookrightarrow \mathbb{C}$ into the complex numbers, so that we are in the situation of a connection on a curve over $\mathbb{C}$. Uniqueness of formal solutions show that the $u_{w}$ are just the power series expansions of analytic solutions near $b$. But the analytic solutions can be obtained as iterated integrals: in a contractible neighborhood of $b$, choose a path $c$ from $b$ to $x$. Then for $w=A_{i_{1}} A_{i_{2}} \cdots A_{i_{n}}$, we have

$$
u_{w}=\int_{c} \alpha_{i_{1}} \alpha_{i_{2}} \cdots \alpha_{i_{n}}
$$

We see therefore, that $u(x)$ can also be continued as a multi-valued function to all of $X_{v}$ (which, recall, we are now regarding as a complex curve). Regard the $u_{w}$ as functions on the universal covering $\tilde{X}_{v}$ after choosing a point $b^{\prime} \in \tilde{X}_{v}$ lying over $b$. Let the topological fundamental group $\pi$ of $X_{v}$ act this time on the space of analytic functions on $\tilde{X}_{v}$ by $\gamma: f(z) \mapsto f^{\gamma}(z)=f(z \gamma)$. This extends to an action of the complex group algebra $\mathbb{C}[\pi]$. To compute $u_{w}^{\gamma}(z)$ for an element $\gamma \in \pi$, we proceed as follows: Choose a path $c$ from $b^{\prime} \gamma$ to $z$. Now choose a lifting $\tilde{\gamma}$ of $\gamma$ to a path in $\tilde{X}_{v}$ from $b^{\prime}$ to $b^{\prime} \gamma$. Then

$$
\begin{aligned}
u_{w}(z \gamma)=\int_{c \tilde{\gamma}} \alpha_{i_{1}} \cdots \alpha_{i_{n}}= & u_{w}(z)+\int_{c} \alpha_{i_{1}} \cdots \alpha_{i_{n-1}} \int_{\tilde{\gamma}} \alpha_{i_{n}} \\
& +\int_{c} \alpha_{i_{1}} \cdots \alpha_{i_{n-2}} \int_{\tilde{\gamma}} \alpha_{i_{n-1}} \alpha_{i_{n-2}}+\cdots
\end{aligned}
$$

As before, let $a_{i} \in \mathbb{C}[\pi]$ be elements whose image in $H_{1}\left(X_{v}, \mathbb{C}\right)$ form a basis dual to the $\alpha_{i}$. Then we see that

$$
u_{w A_{i}}^{a_{i}}=\operatorname{deg}\left(a_{i}\right) u_{w A_{i}}+u_{w}+\Sigma_{\left|w^{\prime}\right|<|w|} c_{w^{\prime}} u_{w^{\prime}}
$$

for constants $c_{w^{\prime}}$.

Now we prove the desired independence by induction on $|w|$. So assume we are done for $|w|<n$, and suppose we have a linear relation

$$
\Sigma_{|w| \leq n} h_{w} u_{w}=0
$$


where the $h_{w}$ are algebraic. In particular, they are single-valued on $X_{v}$, and so are acted on trivially by $\pi$. We write this relation as

$$
\Sigma_{|w|=n} h_{w} u_{w}+\Sigma_{|w|=n-1} h_{w} u_{w}+\Sigma_{|w| \leq n-2} h_{w} u_{w}=0
$$

or

$$
\Sigma_{i}\left[\Sigma_{|w|=n-1} h_{w A_{i}} u_{w A_{i}}+\Sigma_{|w|=n-2} h_{w A_{i}} u_{w A_{i}}\right]+\Sigma_{|w| \leq n-2} h_{w} u_{w}=0
$$

When we apply $a_{j}-\operatorname{deg}\left(a_{j}\right) I$ to this relation, we get

$$
\Sigma_{|w|=n-1} h_{w A_{j}} u_{w}+\Sigma_{|w| \leq n-2} b_{w} u_{w}=0
$$

for some algebraic functions $b_{w}$. By induction, we get $h_{w A_{j}}=0$ for all $w$. Since this works for any $j$, we are done.

\section{§2. Étale Realizations}

This time we start with a smooth scheme $X$ over a field $L$ of characteristic zero and put $\bar{X}=X \otimes_{L} \bar{L}$. We have on $\bar{X}$ the category $\operatorname{Un}_{p}^{e t}(\bar{X})$ of unipotent $\mathbb{Q}_{p}$-lisse sheaves. Here, the unipotence refers to the existence of a filtration by sub-lisse-sheaves such that the associated graded objects are direct sums of the trivial $\mathbb{Q}_{p}$-sheaf. Choosing a point $b \in \bar{X}$ then determines a fiber functor $e_{b}: \operatorname{Un}_{p}^{e t}(X) \rightarrow \operatorname{Vect}_{\mathbb{Q}_{p}}$ by taking stalks. We define the pro-unipotent $p$-adic étale fundamental group (see, e.g., [31]) and path space by

$$
\pi_{1, \text { ét }}(\bar{X}, b)=\operatorname{Aut}^{\otimes}\left(e_{b}\right)
$$

and

$$
\pi_{1, \text { ét }}(\bar{X} ; x, b)=\operatorname{Isom}^{\otimes}\left(e_{b}, e_{x}\right),
$$

where, as usual, we have represented an obvious functor on $\mathbb{Q}_{p}$ algebras. When both $b$ and $x$ are in $X(L)$, we get an action of $G=\operatorname{Gal}(\bar{L} / L)$. In particular, $P^{e t}(x)=\pi_{1 \text {,ét }}(\bar{X} ; x, b)=\operatorname{Spec}\left(\mathcal{P}^{e t}\right)$ is a right $U^{e t}=\pi_{1 \text {,ét }}(\bar{X}, b)=\operatorname{Spec}\left(A^{e t}\right)$ torsor in a manner compatible with the Galois action. Here as well, we can consider the subcategory $\operatorname{Un}_{p, n}^{e t}(\bar{X})$ of unipotent $\mathbb{Q}_{p}$ local systems $V$ having index of unipotency $\leq n$ in that $V$ is endowed with a filtration

$$
V=V_{n} \supset V_{n-1} \supset \cdots \supset V_{1} \supset V_{0}=0
$$

such that each successively quotient is isomorphic to a trivial $\mathbb{Q}_{p}$-sheaf. The corresponding restrictions of the fiber functors will again be denoted $e_{b}^{n}$, etc. 
We denote by $<\operatorname{Un}_{p, n}^{e t}(\bar{X})>$ the Tannakian subcategory of $\operatorname{Un}_{p}^{e t}(\bar{X})$ generated by $\operatorname{Un}_{p, n}^{e t}(\bar{X})$. Using the restrictions $\left\langle e_{b}^{n}>:=e_{b}\right|<\operatorname{Un}_{p, n}^{e t}(\bar{X})>$ and $\left\langle e_{x}^{n}>:=\right.$ $e_{x} \mid<\operatorname{Un}_{p, n}^{e t}(\bar{X})>$ of the fiber functors, we then get $U_{n}^{e t}$ and $P_{n}^{e t}(x)$. In the above, we can everywhere consider homomorphisms of functors rather than tensor isomorphisms, like $\operatorname{Hom}\left(e_{b}, e_{x}\right)$, which of course have the structure of $\mathbb{Q}_{p}$ vector spaces.

It might be useful to recall at this point some rudimentary points about the structure of these groups and torsors, although it is not necessary to be as explicit as in the De Rham case.

Let $E^{e t}$ be the universal enveloping algebra of Lie $U^{e t}$. Then $E^{e t}$ again has the structure of a co-commutative Hopf algebra, and we define $E^{e t}[n]:=$ $E^{e t} / I^{n}$, where $I \subset E$ is the kernel of the counit $E^{e t} \rightarrow \mathbb{Q}_{p}$. As in the De Rham situation, we have the universal pro-unipotent lisse $\mathbb{Q}_{p}$-sheaf $\mathcal{E}^{e t}$ that can be constructed, for example, by twisting the canonical profinite torsor $\hat{T}:=P^{\wedge} \mid X \times b$, in the notation of [10], section 10.17, with representation of the profinite $\hat{\pi}_{1}(\bar{X}, b)$ obtained by composing the natural map

$$
\hat{\pi}_{1}(\bar{X}, b) \rightarrow U^{e t}
$$

with the left multiplication of $U^{e t}$ on $E$. By an argument identical to the De Rham setting, we deduce the property that $\mathcal{E}_{x}^{e t}$ is canonically isomorphic to $\operatorname{Hom}\left(e_{b}, e_{x}\right)$ and the finite-rank quotients $\mathcal{E}^{e t}[n]$ corresponding to $E / I^{n}$ are isomorphic in their turn $\operatorname{Hom}\left(e_{b}^{n}, e_{x}^{n}\right)$. Once again the universal property gives us a comultiplication

$$
\Delta: \mathcal{E}^{e t} \rightarrow \mathcal{E}^{e t} \otimes \mathcal{E}^{e t}
$$

(here and henceforward, a tensor product without an explicit subscript will refer to tensoring over $\left.\mathbb{Q}_{p}\right)$ so that if we define $\mathcal{P}^{e t}=\operatorname{Hom}\left(\mathcal{E}^{e t}, \mathbb{Q}_{p}\right)$, then $\mathcal{P}^{e t}$ is an ind- $\mathbb{Q}_{p}$ lisse sheaf with the property that $\mathcal{P}_{x}^{e t}$ is the coordinate ring of $P^{e t}(x)$. There is also a parallel (to the De Rham setting) discussion corresponding to $\mathcal{P}^{e t}[n], \mathcal{P}_{n}^{e t}$, and $P_{n}^{e t}$, which we will assume without further comment. We will refer again to the filtration by the $\mathcal{P}^{e t}[n]$ as the Eilenberg-Maclane filtration. When $x \in X(L)$, since the categories $\operatorname{Un}_{p . n}^{e t}(\bar{X})$ are invariant under the Galois action, so is $\mathcal{P}^{e t}[n](x)$. Therefore, $\mathcal{P}^{e t}$ is a direct limit of the finite-dimensional Galois modules $\mathcal{P}^{e t}[n](x)$, allowing much of the theory of finite-dimensional representations, for example Fontaine's theory of the Dieudonné functor [12], to apply.

In the subsequent discussion, fix a quotient group $G$ through which the action of $\operatorname{Gal}(\bar{L} / L)$ factors.

We recall some notions from [23]. Given a $\mathbb{Q}_{p}$-algebra $R$, we give it 
the inductive limit topology obtained from the natural topology on finitedimensional $\mathbb{Q}_{p}$-subspaces. Then by a torsor for $U^{e t}$ over $R$, we mean a $U_{R}^{e t}$ torsor $T=\operatorname{Spec}(\mathcal{T})$ in the usual sense, except that we require that

(1) $\mathcal{T}=\varliminf_{\longrightarrow} \mathcal{T}[n]$ for some locally free sub- $R$-modules $\mathcal{T}[n]$ that are local direct summands and of finite rank equal to $\operatorname{dim} A^{e t}[n]$, as $n$ runs through the non-negative integers.

(2) $\mathcal{T}$ is equipped with a continuous $R$-linear action of $G$ that stabilizes the $\mathcal{T}[n]$.

(3) The torsor structure

$$
\mathcal{T} \rightarrow \mathcal{T} \otimes_{R}\left(A^{e t} \otimes R\right)
$$

is compatible with the $G$-action and takes $\mathcal{T}[n]$ to $\mathcal{T}[n] \otimes_{R}\left(A^{e t}[n] \otimes R\right)$.

When $L$ is the completion $F_{v}$ of a number field $F$ as in the introduction and $G=G_{v}=\operatorname{Gal}\left(F_{v} / F\right)$, we get a classifying pro-variety $H^{1}\left(G_{v}, U^{e t}\right)$ [23] defined over $\mathbb{Q}_{p}$ for torsors, as well as a subvariety $H_{g}^{1}\left(G_{v}, U^{e t}\right)$, consisting of those torsors that trivialize upon base-change to $B_{D R}$. We warn the reader at this point that this subvariety was denoted $H_{f}^{1}$ in the reference [23]. In this paper, we will use the notation $H_{f}^{1}$ for a certain subvariety of $H_{g}^{1}$ defined using $B_{c r}$ in place of $B_{D R}$ (see below). Let us spell out what this $B_{D R}$ condition means. If $T=\operatorname{Spec}(\mathcal{T})$ is a torsor for $U^{e t}$ over $R$, we say it is a De Rham torsor if there is a $G_{v}$-equivariant algebra homomorphism

$$
t: \mathcal{T} \rightarrow R \otimes B_{D R}
$$

or equivalently,

$$
t_{B_{D R}}: \mathcal{T} \otimes B_{D R} \rightarrow R \otimes B_{D R} .
$$

That is, we are requiring the existence of a $G_{v}$-invariant point in $T_{B_{D R}}(R \otimes$ $\left.B_{D R}\right)$. Then $t$ induces an isomorphism of $B_{D R^{-}}$algebras

$$
C_{t}: \mathcal{T} \otimes B_{D R} \simeq A^{e t} \otimes R \otimes B_{D R}
$$

that is $G_{v}$-equivariant. Furthermore, since $t_{B_{D R}}$ is a homomorphism of $B_{D R}$ algebras, it respects the Hodge filtration induced by that on $B_{D R}$. Hence, so does $C_{t}$. Since there is the identity homomorphism

$$
e: A^{e t} \otimes R \otimes B_{D R} \rightarrow R \otimes B_{D R},
$$

out of which we recover $t$ as $e \circ C_{t}$, we see that the datum of $C_{t}$ is equivalent to the De Rham condition. 
Taking $G_{v}$-invariants, we get an isomorphism

$$
\begin{aligned}
C_{t^{G_{v}}}: D(\mathcal{T}):=\left(\mathcal{T} \otimes B_{D R}\right)^{G_{v}} & \simeq\left(A^{e t} \otimes B_{D R}\right)^{G_{v}} \otimes R \\
& \simeq A^{D R} \otimes R=A^{D R} \otimes_{F_{v}}\left(F_{v} \otimes R\right)
\end{aligned}
$$

Here, we are using the fact that $D\left(A^{e t}\right) \simeq A_{D R}$ in a manner compatible with the Hodge filtration ([27], [30], [11]). As a consequence of the definition, the coordinate $\operatorname{ring} \mathcal{T}$ becomes a limit of finite-dimensional De Rham representations in the sense of [12] and hence, the inclusion

$$
D(\mathcal{T}) \hookrightarrow \mathcal{T} \otimes B_{D R}
$$

induces an isomorphism

$$
D(\mathcal{T}) \otimes_{F_{v}} B_{D R} \simeq \mathcal{T} \otimes B_{D R}
$$

If we examine what happens to the torsor structure $\mathcal{T} \rightarrow \mathcal{T} \otimes_{R}\left(A^{\text {et }} \otimes R\right)$, it extends to

$$
\mathcal{T} \otimes B^{D R} \rightarrow \mathcal{T} \otimes_{R}\left(A^{e t} \otimes R\right) \otimes B^{D R}
$$

But

$$
\begin{aligned}
& \mathcal{T} \otimes_{R}\left(A^{e t} \otimes R\right) \otimes B^{D R}=\left(\mathcal{T} \otimes A^{e t}\right) \otimes B_{D R}=\left(\mathcal{T} \otimes B_{D R}\right) \otimes_{B_{D R}}\left(A^{e t} \otimes B_{D R}\right) \\
& \quad=\left(\left(\mathcal{T} \otimes B_{D R}\right)^{G_{v}} \otimes_{F_{v}} B_{D R}\right) \otimes_{B_{D R}}\left(\left(A^{e t} \otimes B_{D R}\right)^{G_{v}} \otimes_{F_{v}} B_{D R}\right) \\
& \quad=D(\mathcal{T}) \otimes_{F_{v}} D\left(A^{e t}\right) \otimes B_{D R}
\end{aligned}
$$

So taking $G_{v}$-invariants gives us

$$
D(\mathcal{T}) \rightarrow D(\mathcal{T}) \otimes_{F_{v}} A^{D R}=D(\mathcal{T}) \otimes_{R \otimes F_{v}}\left(A^{D R} \otimes_{F_{v}}\left(R \otimes F_{v}\right)\right) .
$$

This defines the structure of a $U^{D R}$-torsor over $R \otimes F_{v}$ on $D(T)$ which is trivialized by the point $t^{G_{v}}: D(\mathcal{T}) \rightarrow R \otimes F_{v}$ obtained from $t$ by restricting to the $G_{v}$-invariants. In fact, the map

$$
D(\mathcal{T}) \rightarrow A^{D R} \otimes_{F_{v}}\left(R \otimes F_{v}\right)
$$

obtained from $t^{G_{v}}$ is seen to be none other than the earlier isomorphism $C_{t^{G_{v}}}$. The compatibility of the torsor structure with the Eilenberg-Maclane filtration follows from the fact that the comparison isomorphism of [27] arises from an equivalence of categories

$$
\mathrm{Un}_{p}^{e t}(\bar{X}) \otimes B_{D R} \simeq \mathrm{Un}^{D R}(X) \otimes_{F_{v}} B_{D R}
$$


that respects the subcategories specified by the index of unipotency on either side.

The Hodge filtration on $D(\mathcal{T})$ is defined to be the one induced by the Hodge filtration on $B_{D R}$. Since this is true also of the one on $A^{D R}=D\left(A^{\text {et }}\right)$ and $\mathcal{T} \otimes B_{D R} \simeq A^{e t} \otimes R \otimes B_{D R}$ preserves the filtration, so does $D(\mathcal{T}) \simeq A^{D R} \otimes_{F_{v}} R$. We conclude that the $U^{D R}$ torsor thus obtained can be trivialized together with the Hodge filtration, in the sense of the previous section.

There is a parallel discussion with $B_{c r}$ in place of $B_{D R}$ that defines a crystalline $U^{e t}$-torsor $T$ over $R$ to be one that trivializes over $B_{c r}$ or, equivalently, admits a $G_{v}$-equivariant isomorphism of torsors

$$
\mathcal{T} \otimes B_{c r} \simeq A^{e t} \otimes B_{c r}
$$

Using the usual inclusion of $B_{c r}$ into $B_{D R}$, we see that a crystalline torsor is also De Rham. It is easy to show that the crystalline condition defines a subvariety

$$
H_{f}^{1}\left(G_{v}, U^{e t}\right) \subset H_{g}^{1}\left(G_{v}, U^{e t}\right)
$$

However, since the reference [23] dealt explicitly only with $H_{g}$, we sketch the modification necessary to represent the crystalline torsors. For each $n$, consider the functor on $\mathbb{Q}_{p}$-algebras

$$
H^{0}\left(G_{v}, U_{n}^{e t, B_{c r}} / U_{n}^{e t}\right): R \mapsto H^{0}\left(G_{v}, U_{n}^{e t}\left(B_{c r} \otimes R\right) / U_{n}^{e t}(R)\right)
$$

Then we have

Lemma 5. $H^{0}\left(G_{v}, U_{n}^{e t, B_{c r}} / U_{n}^{e t}\right)$ is representable by an affine variety over $\mathbb{Q}_{p}$.

The proof is verbatim the same as [23], section 1, proposition 3 and will be therefore omitted. And then, as in the few paragraphs preceding that proposition, we have an exact sequence

$$
0 \rightarrow U_{n}^{e t}(R) \rightarrow U_{n}^{e t}\left(R \otimes B_{c r}\right) \rightarrow U_{n}^{e t}\left(R \otimes B_{c r}\right) / U_{n}^{e t}(R) \rightarrow 0
$$

from which we get the connecting homomorphism

$$
H^{0}\left(G_{v}, U_{n}^{e t}\left(R \otimes B_{c r}\right) / U_{n}^{e t}(R)\right) \rightarrow H^{1}\left(G_{v}, U_{n}^{e t}(R)\right)
$$

which is functorial in $R$. Thus, it is represented by a map of varieties, and its image is a subvariety of $H^{1}\left(U_{n}^{e t}(R)\right)$. But by looking at the crystalline condition as the existence of a $G_{v}$-invariant point in $P^{e t}\left(R \otimes B_{c r}\right)$, we see that 
the image consists exactly of the crystalline torsors (exactly as in the proof of [23], section 2, lemma 6). By passing to the limit over $n$, we see that the set of isomorphisms classes of crystalline torsors for $U^{\text {et }}$ is represented by a pro-algebraic subvariety $H_{f}^{1}\left(G_{v}, U^{e t}\right)$ of $H_{g}^{1}\left(G_{v}, U^{e t}\right)$.

If we denote by $K$ the absolutely unramified subfield of $F_{v}$, then we get a functor $D_{c r}$ from crystalline $U^{e t}$-torsors over $R$ to (trivial) $U^{c r}$-torsors over $R \otimes K$ endowed with a Frobenius endomorphism: This functor of course sends $T=\operatorname{Spec}(\mathcal{T})$ to

$$
D_{c r}(T):=\operatorname{Spec}\left(\left(\mathcal{T} \otimes B_{c r}\right)^{G_{v}}\right) .
$$

By the comparison isomorphism of [27], a discussion entirely parallel to the $B_{D R}$ case shows that $D_{c r}(T)$ is endowed with a Frobenius endomorphism and is a torsor for $U^{c r}=D_{c r}\left(U^{e t}\right)$ in a manner compatible with this extra structure. As always, this torsor is canonically trivial. We have the comparison

$$
D(T) \simeq D_{c r}(T) \otimes_{K} F_{v} .
$$

Thereby, for crystalline torsors $T, D(T)$ is endowed with both a Hodge filtration and a Frobenius.

To summarize, the functor $T \mapsto D(T)$ defines a map from the isomorphism classes of crystalline $U^{e t}$-torsors over $R$ to admissible $U^{D R}$ torsors over $R \otimes F_{v}$. It is therefore represented by a pro-algebraic map

$$
D: H_{f}^{1}\left(G_{v}, U^{e t}\right) \rightarrow \operatorname{Res}_{\mathbb{Q}_{p}}^{F_{v}}\left(U^{D R} / F^{0}\right) .
$$

Going back from abstract considerations to the study of rational points, each $P^{e t}(x)$ defines a class in $H_{f}^{1}\left(G_{v}, U^{e t}\right)$ [27]. We are thus given an étale unipotent Albanese map

$$
j_{l o c}^{e t}: x \mapsto\left[P^{e t}(x)\right] \in H_{f}^{1}\left(G_{v}, U^{e t}\right) .
$$

When we let $L=F$, the number field itself and let $b \in \mathcal{X}(R)$ be an integral point, then the $\Gamma=\operatorname{Gal}(\bar{F} / F)$-action factors through $G=G_{T}$ (as in [31], proof of theorem 2.8), with notation following the introduction. Here $R$ and $T$ are as in the introduction. As explained in loc. cit., the point here is that there is an exact sequence

$$
0 \rightarrow U^{e t} \rightarrow U^{e t, a r} \rightarrow G_{T} \rightarrow 0
$$

that is split by the base-point $b$, accounting for the $\Gamma$ action on $U^{e t}$ and also for the twisted actions corresponding to other points. (Here, the middle term is an 'arithmetic' fundamental group obtained by pushing out a pro-finite arithmetic 
fundamental group $\hat{\pi}_{1}(\mathcal{X}, b)$ using $U^{e t}$.) Therefore, we have a global continuous cohomology pro-variety

$$
H^{1}\left(G_{T}, U^{e t}\right)
$$

and a classifying sub-variety

$$
H_{f}^{1}\left(G_{T}, U^{e t}\right) \subset H^{1}\left(G_{T}, U^{e t}\right),
$$

defined to be the inverse image of $H_{f}^{1}\left(G_{v}, U^{e t}\right)$ under the restriction map

$$
H^{1}\left(G_{T}, U^{e t}\right) \rightarrow H^{1}\left(G_{v}, U^{e t}\right) .
$$

Using this environment, we can finally define the global unipotent Albanese map

$$
j_{\text {glob }}^{e t}: x \in \mathcal{X}(R) \mapsto\left[P^{e t}(x)\right] \in H_{f}^{1}\left(G_{T}, U^{e t}\right) .
$$

To conclude, we have constructed a commutative diagram

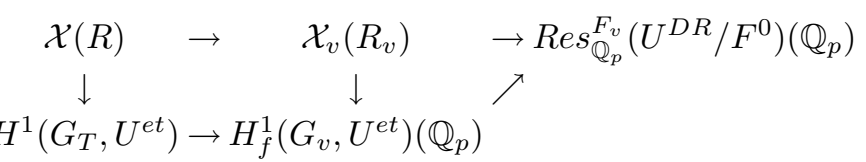

where the commutativity of the triangle on the right is in [11] and [27], following earlier cases of [28], [30]. The key point here is the comparison isomorphism:

$$
\pi_{1}^{D R}\left(X_{v} ; b, x\right) \otimes B_{c r} \simeq \pi_{1, \text { ét }}\left(\bar{X}_{v} ; b, x\right) \otimes B_{c r}
$$

that respects the action of $G_{v}$ as well as the Hodge filtration on Frobenius action.

Passing from here to the finite-level quotients for each $n$ gives us the diagram from the introduction:

$$
\begin{array}{cccc}
\mathcal{X}(R) & \rightarrow & \mathcal{X}_{v}\left(R_{v}\right) & \rightarrow \\
\downarrow & & \operatorname{Res}_{\mathbb{Q}_{p}}^{F_{v}}\left(U_{n}^{D R} / F^{0}\right)\left(\mathbb{Q}_{p}\right) \\
H^{1}\left(G_{T}, U_{n}^{e t}\right) & \rightarrow H^{1}\left(G_{v}, U_{n}^{e t}\right)\left(\mathbb{Q}_{p}\right) & \nearrow
\end{array}
$$

\section{$\S 3 . \quad$ Comments I}

The proof of the statement that conjecture 1 implies the finiteness of $\mathcal{X}(R)$ is exactly as in [23] and in Chabauty's original argument. The dimension assumption implies that for $n$ as in the conjecture, the image of the map

$$
H_{f}^{1}\left(G_{T}, U_{n}^{e t}\right) \rightarrow U_{n}^{D R} / F^{0}
$$


is contained in a proper Zariski-closed subset. Hence, so is the image of

$$
\mathcal{X}(R) \rightarrow U_{n}^{D R} / F^{0} .
$$

We deduce that there is a non-zero algebraic function on $U_{n}^{e t} / F^{0}$ that vanishes on this image. But by theorem 1, the pull-back of this function is not identically zero on any residue disk of $\mathcal{X}\left(R_{v}\right)$ and is a Coleman function [2]. Therefore, its zero set on $\mathcal{X}\left(R_{v}\right)$ is finite. It is perhaps worth noting that $\mathcal{X}\left(R_{v}\right) \rightarrow U_{n}^{D R} / F^{0}$ is at most finite-to-one (this follows from the $n=2$ case). The essential point we have shown then is that

$$
\operatorname{Im}(\mathcal{X}(R)) \subset \operatorname{Im}\left(\mathcal{X}\left(R_{v}\right)\right) \cap \operatorname{Im}\left(H_{f}^{1}\left(G_{T}, U_{n}^{e t}\right)\right)
$$

and that the latter intersection is finite (of course assuming conjecture 1). Therefore, the strategy of this paper is reminiscent of Serge Lang's suggestion [25] to prove directly that the intersection of a curve with the Mordell-Weil group of its Jacobian is finite, except that the approach here is p-adic and non-abelian.

We proceed to comment on the relation between conjecture 1 and various standard conjectures. Such a connection is based upon a study of various groups of Selmer type. Given a $\mathbb{Q}_{p}$-representation $V$ of $G_{T}$ we will be especially interested in

$$
S e l_{T}^{0}(V):=\operatorname{Ker}\left(H^{1}\left(G_{T}, V\right) \rightarrow \oplus_{w \in T} H^{1}\left(G_{w}, V\right)\right)
$$

Conjecture 2. Let $X / F$ be a smooth curve and let $V_{n}=H_{e t}^{1}(\bar{X}$, $\left.\mathbb{Q}_{p}\right)^{\otimes n}(1)$. Then $\operatorname{Sel}_{T}^{0}\left(V_{n}\right)=0$ for $n>>0$.

This conjecture appears to be a special case of some general expectations about motivic representations, and I am not sure to whom it should be attributed. Notice that $V_{n}$ is a direct summand of $H^{n}\left(\bar{X}^{n}, \mathbb{Q}_{p}(1)\right)$, by the Künneth formula. There is are maps from $K$-theory ([3], section 5 )

$$
c h_{n, r}: K_{2 r-n-1}^{(r)}\left(X^{n}\right) \rightarrow H^{1}\left(\operatorname{Gal}(\bar{F} / F), H^{n}\left(\bar{X}^{n}, \mathbb{Q}_{p}(r)\right)\right)
$$

for $2 r-1 \neq n$, where the superscript in the $K$-group refers to an associated graded space for the gamma filtration. The images of the $c h_{n, r}$ lie inside the subspaces $H_{g}^{1}\left(\operatorname{Gal}(\bar{F} / F), H^{n}\left(\bar{X}^{n}, \mathbb{Q}_{p}(r)\right)\right)$ consisting of cohomology classes that are unramified at almost all primes and potentially semi-stable at $p$. In any case,

$$
\operatorname{Sel}_{T}^{0}\left(V_{n}\right) \subset H_{g}^{1}\left(\operatorname{Gal}(\bar{F} / F), H^{n}\left(\bar{X}^{n}, \mathbb{Q}_{p}(1)\right)\right) .
$$


In the course of formulating their famous conjectures, Bloch and Kato conjectured ([3], conjecture 5.3) that $c h_{n, r}$ induces an isomorphism

$$
K_{2 r-n-1}^{(r)}\left(X^{n}\right) \otimes \mathbb{Q}_{p} \simeq H_{g}^{1}\left(\operatorname{Gal}(\bar{F} / F), H^{n}\left(\bar{X}^{n}, \mathbb{Q}_{p}(r)\right)\right)
$$

It is also stating that certain extensions are geometric in a specific sense. Of course, the section conjecture can also be viewed in a similar light. In any case, if $n>2 r-1$ the $K$-groups are zero. Therefore

Observation 1. The conjecture of Bloch and Kato implies conjecture 2.

Tony Scholl has pointed out to me that

Observation 2. Conjecture 2 is also implied by the Fontaine-Mazur conjecture [13].

Recall that the Fontaine-Mazur conjecture proposes that a continuous irreducible $\mathbb{Q}_{p}$-representation of $\operatorname{Gal}(\overline{\mathbb{Q}} / \mathbb{Q})$ that is unramified at almost all places and potentially semi-stable at $p$ is motivic. An extension of two such is then conjectured to be a $\mathbb{Q}_{p}$-linear combination of motivic representations in a suitable Ext group. Hence, an extension

$$
0 \rightarrow V_{n} \rightarrow E \rightarrow \mathbb{Q}_{p} \rightarrow 0
$$

corresponding to an element of $\operatorname{Sel}_{T}^{0}\left(V_{n}\right)$ must be a $\mathbb{Q}_{p}$ linear combination of extensions of geometric origin. In particular, it must carry a weight filtration. $V_{n}$ is pure of weight $n-2$. Since a map between mixed representations is strict for the weight filtration, there exists a vector $v \in W_{0} E$ that maps to $1 \in \mathbb{Q}_{p}$. If $n \geq 3$, then $W_{0} E \cap V_{n}=0$, and hence, $W_{0} E$ must be a 1-dimensional complement to $V_{n}$. Thus, the extension must split.

We will show

Proposition 2. Conjecture 2 implies conjecture 1.

If we use the symbol $U^{M}$ for the fundamental group in any of the realizations of interest and $U_{n}^{M}=Z^{n} \backslash U^{M}$, then we have an exact sequence

$$
0 \rightarrow Z^{n+1} \backslash Z^{n} \rightarrow U_{n+1}^{M} \rightarrow U_{n}^{M} \rightarrow 0
$$

We need to calculate the dimension $d_{n}$ of $Z^{n+1} \backslash Z^{n}$. This is achieved as follows: $U^{M}$ is the unipotent completion of either a free group on $m=2 g+s-1$ generators, where $g$ is the genus of $X^{\prime}$ and $s$ the order of $X^{\prime} \backslash X$, or a free 
group on $2 g$ generators modulo a single relation of degree 2 (the compact case). According to [24] $d_{n}$ is given by the recursive formula

$$
\Sigma_{k \mid n} k d_{k}=m^{n}
$$

in the open case and

$$
\Sigma_{k \mid n} k d_{k}=\left(g+\sqrt{g^{2}-1}\right)^{n}+\left(g-\sqrt{g^{2}-1}\right)^{n}
$$

in the compact case. So one gets the asymptotics $d_{n} \approx m^{n} / n$ in the noncompact case and $d_{n} \approx\left(g+\sqrt{g^{2}-1}\right)^{n} / n$ in the compact case. (The small difference between the two formulas appears to be significant. Even after considerable effort, the argument of observation 3 below could not be adapted to the compact case.) Notice here that in the compact case, $g \geq 2$. We can also estimate $F^{0}\left(Z^{n+1} \backslash Z^{n}\right)$ in the De Rham realization by noting that it is a quotient of $H_{1}^{D R}\left(X_{v}\right)^{\otimes n}$ which has the filtration dual to that on $H_{D R}^{1}\left(X_{v}\right)^{\otimes n}$. So $F^{0} H_{1}^{D R}\left(X_{v}\right)^{\otimes n}$ has dimension equal to the codimension of

$$
\begin{aligned}
& F^{1} H_{D R}^{1}\left(X_{v}\right)^{\otimes n} \\
= & {\left[F^{1} H_{D R}^{1} \otimes\left(H_{D R}^{1}\right)^{\otimes(n-1)}\right] \oplus\left[H_{D R}^{1} \otimes F^{1} H_{D R}^{1} \otimes\left(H_{D R}^{1}\right)^{\otimes(n-2)}\right] \oplus \cdots } \\
& \oplus\left[\left(H_{D R}^{1}\right)^{\otimes(n-1)} \otimes F^{1} H_{D R}^{1}\right]
\end{aligned}
$$

which therefore is equal to

$$
\operatorname{dim} H^{1}\left(\mathcal{O}_{X_{v}^{\prime}}\right)^{\otimes n}=g^{n} .
$$

Therefore, $F^{0}\left(Z^{n+1} \backslash Z^{n}\right) \leq g^{n}$. In particular, it does not contribute to the asymptotics. That is, the jump in dimensions as one goes from $U_{n}^{D R}$ to $U_{n+1}^{D R}$ is determined by the asymptotics of $d_{n}$. On the other hand, in the étale realization, we have

$$
0 \rightarrow H^{1}\left(G_{T}, Z^{n+1} \backslash Z^{n}\right) \rightarrow H^{1}\left(G_{T}, U_{n+1}^{e t}\right) \rightarrow H^{1}\left(G_{T}, U_{n}^{e t}\right)
$$

in the sense of [23] section 1 , whereby the middle term is an $H^{1}\left(G_{T}, Z^{n+1} \backslash Z^{n}\right)$ torsor over a subvariety of the last term. So we can control the change in dimensions using the Euler characteristic formula

$$
\operatorname{dim} H^{1}\left(G_{T}, Z^{n+1} \backslash Z^{n}\right)-\operatorname{dim} H^{2}\left(G_{T}, Z^{n+1} \backslash Z^{n}\right)=\operatorname{dim}\left(Z^{n+1} \backslash Z^{n}\right)^{-},
$$

where the negative superscript refers to the (-1) eigenspace of complex conjugation. By comparison with complex Hodge theory, we see that the right hand side is $d_{n} / 2$ for $n$ odd. So it remains to observe that 
Lemma 6. Conjecture 2 implies

$$
\operatorname{dim} H^{2}\left(G_{T}, Z^{n+1} \backslash Z^{n}\right) \leq P(n) g^{n}
$$

for an effective polynomial $P(n)$ of $n$.

Proof of lemma. Since the $p$-cohomological dimension of $G_{T}$ is 2 [26], we have a surjection

$$
H^{2}\left(G_{T}, H_{1}\left(\bar{X}, \mathbb{Q}_{p}\right)^{\otimes n}\right) \rightarrow H^{2}\left(G_{T}, Z^{n+1} \backslash Z^{n}\right) \rightarrow 0,
$$

so it suffices to prove the estimate for the first group. But by conjecture 2 and Poitou-Tate duality, we get an injection

$$
H^{2}\left(G_{T}, H_{1}\left(\bar{X}, \mathbb{Q}_{p}\right)^{\otimes n}\right) \hookrightarrow \oplus_{w \in T} H^{2}\left(G_{w}, H_{1}\left(\bar{X}, \mathbb{Q}_{p}\right)^{\otimes n}\right)
$$

for $n$ sufficiently large. Hence, we need only estimate the dimension of the local groups. Applying local duality allows us to reduce this to the study of

$$
H^{0}\left(G_{w}, H^{1}\left(\bar{X}, \mathbb{Q}_{p}\right)^{\otimes n}(1)\right) .
$$

For $w$ not dividing $p$, we have a filtration

$$
W: \quad W_{0} \subset W_{1} \subset W_{2}=H^{1}\left(\bar{X}, \mathbb{Q}_{p}\right)
$$

coming (after a finite base-change corresponding to semi-stable reduction) from the theory of Raynaud's universal covering

$$
0 \rightarrow \Gamma \rightarrow G \rightarrow J_{X} \rightarrow 0
$$

where $G$ is an extension of an abelian variety $B$ by a torus $T$. That is, we have a sequence of inclusions

$$
T_{l}(T) \hookrightarrow T_{l}(G) \hookrightarrow T_{l}\left(J_{X}\right)
$$

whose dual gives us the filtration $W$ ([8], section 2). Because $W_{0} \simeq G r_{2}^{W}(-1)$, we have $\operatorname{dim} W_{0}=\operatorname{dim} G r_{2}^{W} \leq g$. From this, we get immediately that

$$
\operatorname{dim} H^{0}\left(G_{w}, H^{1}\left(\bar{X}, \mathbb{Q}_{p}\right)^{\otimes n}(1)\right) \leq n \operatorname{dim} G r_{2}^{W} g^{n-1}+\frac{n(n-1)}{2}\left(\operatorname{dim} G r_{1}^{W}\right)^{2} g^{n-2}
$$

For $w$ dividing $p$, we use the Hodge-Tate decomposition:

$$
H^{1}\left(\bar{X}, \mathbb{Q}_{p}\right) \otimes \mathbb{C}_{p} \simeq H^{0}\left(X^{\prime}, \Omega_{X^{\prime}}(\log D)\right) \otimes \mathbb{C}_{p}(-1) \oplus H^{1}\left(X^{\prime}, \mathcal{O}_{X^{\prime}}\right) \otimes \mathbb{C}_{p}
$$


as $G_{w}$-representations. Thus,

$$
\begin{aligned}
& H^{0}\left(G_{w}, H^{1}\left(\bar{X}, \mathbb{Q}_{p}\right)^{\otimes n}(1)\right) \\
& \quad \hookrightarrow H^{0}\left(G_{w},\left[H^{0}\left(X^{\prime}, \Omega_{X^{\prime}}(\log D)\right) \otimes \mathbb{C}_{p}(-1) \oplus H^{1}\left(X^{\prime}, \mathcal{O}_{X^{\prime}}\right) \otimes \mathbb{C}_{p}\right]^{\otimes n}(1)\right)
\end{aligned}
$$

from which we easily extract the estimate

$$
\operatorname{dim} H^{0}\left(G_{w}, H^{1}\left(\bar{X}, \mathbb{Q}_{p}\right)^{\otimes n}(1)\right) \leq n(g+s-1) g^{n-1}
$$

Since we have already discussed the structure of $U^{M}$ in the proof of the previous proposition, it becomes easy to outline the relationship to a conjecture of Jannsen. There, one starts with any smooth projective variety $V$ over $F$ having good reduction outside a finite set $T$ of primes (which we take to include all primes dividing $p)$ and considers $H^{2}\left(G_{T}, H^{n}\left(\bar{V}, \mathbb{Q}_{p}\right)(r)\right)$ with various twists $r$. Then Jannsen conjectures:

$$
H^{2}\left(G_{T}, H^{n}\left(\bar{V}, \mathbb{Q}_{p}\right)(r)\right)=0
$$

for $r \geq n+2$.

In fact, we need only a weaker variant

(Weak Jannsen conjecture) There exists a $k>0$ such that for varieties $V$ as above

$$
H^{2}\left(G_{T}, H^{n}\left(\bar{V}, \mathbb{Q}_{p}\right)(r)\right)=0
$$

for $r \geq n+k$

Observation 3. When $X$ is affine, Jannsen's conjecture in the weaker version implies conjecture 1.

The point here is to try once more to estimate the $H^{2}$. But in the affine case, the growth rate of $Z^{n+1} \backslash Z^{n}$ is like $m^{n} / n$, so it will certainly suffice to show that $H^{2}\left(G_{T}, H_{1}\left(\bar{X}, \mathbb{Q}_{p}\right)^{\otimes n}\right)$ is bounded by $C a^{n}$ for some $a<m$. Now, easy homological arguments give us the existence of a constant $K$ such that

$$
\operatorname{dim} H^{2}\left(G_{T}, V\right) \leq K \operatorname{dim} V
$$

Recall that $m=2 g+s-1$ and that $H_{1}\left(\bar{X}, \mathbb{Q}_{p}\right)$ fits into an exact sequence

$$
0 \rightarrow W \rightarrow H_{1}\left(\bar{X}, \mathbb{Q}_{p}\right) \rightarrow V \rightarrow 0
$$

where $W=\mathbb{Q}_{p}(1)^{s-1}$ and $V=H_{1}\left(\bar{X}^{\prime}, \mathbb{Q}_{p}\right)$. (Here, we have passed to a finite extension where all the points at infinity are rational. This passage is admissible for our $H^{2}$ considerations.) Therefore, in the Grothendieck group, we get

$$
H_{1}\left(\bar{X}, \mathbb{Q}_{p}\right)^{\otimes n}=V^{\otimes n} \oplus n\left(V^{\otimes(n-1)} \otimes W\right) \oplus \cdots \oplus\left(\begin{array}{l}
n \\
k
\end{array}\right)\left(V^{\otimes(n-k)} \otimes W^{k}\right) \oplus \cdots
$$


But $V \simeq H^{1}\left(\bar{X}^{\prime}, \mathbb{Q}_{p}\right)(1)$ so that $V^{\otimes l}$ is a direct summand of $H^{l}\left(\left(\bar{X}^{\prime}\right)^{l}, \mathbb{Q}_{p}\right)(l)$. Therefore, $H^{2}\left(G_{T}, V^{\otimes l}(r)\right)=0$ for $r \geq k$. Hence,

$$
\operatorname{dim} H^{2}\left(G_{T}, H_{1}\left(\bar{X}, \mathbb{Q}_{p}\right)^{\otimes n}\right) \leq P(n) \Sigma_{l=0}^{k-1} \operatorname{dim} H^{2}\left(G_{T}, V^{\otimes(n-l)}(l)\right)
$$

for some polynomial $P(n)$. We analyze the cohomology groups on the right. The Weil pairing induces an inclusion

$$
\mathbb{Q}_{p}(1) \hookrightarrow V^{\otimes 2}
$$

which continues to an inclusion

$$
\mathbb{Q}_{p}(k) \hookrightarrow V^{\otimes(2 k)}
$$

with image a direct summand. Write $V^{\otimes 2 k}=M \oplus \mathbb{Q}_{p}(k)$. On the one hand, since any $M^{\otimes i}$ is a direct summand of a $V^{\otimes l}$, we have

$$
H^{2}\left(G_{T}, V^{\otimes i} \otimes M^{\otimes j}(r)\right)=0
$$

for $r \geq k$. On the other,

$$
V^{\otimes 2 k n}=M^{\otimes n} \oplus M^{\prime}(k)
$$

where $M^{\prime}$ is a direct sum of (non-negative) tensor products of $M$ and $\mathbb{Q}_{p}(1)$. Hence,

$$
V^{\otimes(2 k n+i)}=M^{\otimes n} \otimes V^{\otimes i} \oplus M^{\prime} \otimes V^{\otimes i}(k)
$$

and

$$
H^{2}\left(G_{T}, V^{\otimes(2 k n+i)}(l)\right)=H^{2}\left(G_{T}, M^{\otimes n} \otimes V^{\otimes i}(l)\right)
$$

for $l \geq 0$. Therefore, as $i$ runs through $0, \ldots, 2 k-1$

$$
\begin{gathered}
\operatorname{dim} H^{2}\left(G_{T}, V^{\otimes(2 k n+i)}(l)\right) \leq K \operatorname{dim}(V)^{i} \operatorname{dim} M^{\otimes n} \leq K \operatorname{dim}(V)^{2 k-1}\left((2 g)^{2 k}-1\right)^{n} \\
\leq K \operatorname{dim}(V)^{2 k-1}\left((2 g)^{2 k}-1\right)^{(2 k n+i) /(2 k)} .
\end{gathered}
$$

The point is that as one goes through the range $2 k n$ to $2 k n+(2 k-1)$, the dimension of

$$
\operatorname{dim} H^{2}\left(G_{T}, V^{\otimes(2 k n+i)}(l)\right)
$$

can be bounded by a constant times $(\operatorname{dim} M)^{n}$. Rewriting the exponent, we have that for any $n \geq 0$,

$$
\operatorname{dim} H^{2}\left(G_{T}, V^{\otimes n}(l)\right) \leq K \operatorname{dim}(V)^{2 k-1}\left[\left((2 g)^{2 k}-1\right)^{1 /(2 k)}\right]^{n}
$$

and the desired estimate follows. 


\section{$\S 4 . \quad$ Comments II}

It is rather interesting to consider the special case of an elliptic curve minus the origin. For example, our method shows finiteness of integral points for CMelliptic curves $E$ defined over $\mathbb{Q}$ of rank 1, independently of any conjecture. This follows from analyzing somewhat carefully a refined Selmer variety

$$
\operatorname{Sel}_{T}\left(U_{n}\right),
$$

defined to be the classes in $H_{f}^{1}\left(G_{T}, U_{n}^{e t}\right)$ that are potentially unramified at primes not equal to $p$ and potentially crystalline at $p$. (Here of course, the $U$ refer to the unipotent fundamental groups of $E$ minus the origin.) The definition of this subvariety depends on the construction of certain local subvarieties

$$
H_{p f}^{1}\left(G_{v}, H_{v} ; U_{n}^{e t}\right) \subset H^{1}\left(G_{v}, U_{n}^{e t}\right)
$$

$(v \mid p)$ and

$$
H_{p u n}^{1}\left(G_{v}, H_{v} ; U_{n}^{e t}\right) \subset H^{1}\left(G_{v}, U_{n}^{e t}\right)
$$

( $v$ not dividing $p$ ) for various $v$ and subgroups of finite index $H_{v} \subset G_{v}$. Since the pattern is exactly as in [23], section 1, we will omit the details. The point is that if $N$ is a closed subgroup of $G$, then the restriction map $H^{1}\left(G, U_{n}^{e t}\right) \rightarrow H^{1}\left(N, U_{n}^{e t}\right)$ is functorial. We can therefore construct the unramified cohomology varieties $H_{u n}^{1}\left(G_{v}, U_{n}^{e t}\right)$ as the inverse image of the base-point in $H^{1}\left(I_{v}, U_{n}^{e t}\right)$, where $I_{v} \subset G_{v}$ is the inertia subgroup. For this last object, we need only deal with it when the action of $I_{v}$ on $U_{n}^{e t}$ is trivial. In this case, the cohomology functor is not necessarily representable by a variety. However, we still have functorial sequences

$$
0 \rightarrow H^{1}\left(I_{v}, Z^{n+1} \backslash Z^{n}\right) \rightarrow H^{1}\left(I_{v}, U_{n+1}^{e t}\right) \rightarrow H^{1}\left(I_{v}, U_{n}^{e t}\right)
$$

which are exact in the naive sense that the inverse image of the base point under the second map is the kernel. (However, the other fibers may be empty.) Now, the condition that a class $c \in H^{1}\left(G, U_{n+1}^{e t}\right)$ goes to zero in $H^{1}\left(I_{v}, U_{n+1}^{e t}\right)$ is an intersection of the condition that it goes to zero in $H^{1}\left(I_{v}, U_{n}^{e t}\right)$, which defines a subvariety by induction, and then the condition that the image in $H^{1}\left(I_{v}, Z^{n+1} \backslash Z^{n}\right)$ is trivial. This last space is a vector group, and hence, the resulting condition defines the desired 'unramified' subvariety at level $n+1$.

Then, by considering the restriction maps

$$
r_{v}: H^{1}\left(G_{v}, U_{n}^{e t}\right) \rightarrow H^{1}\left(H_{v}, U_{n}^{e t}\right),
$$


to subgroups of finite index, we get the potentially unramified subvarieties

$$
H_{\text {pun }}^{1}\left(G_{v}, H_{v} ; U_{n}^{e t}\right):=r_{v}^{-1}\left(H_{u n}^{1}\left(H_{v}, U_{n}^{e t}\right)\right)
$$

and potentially crystalline subvarieties

$$
H_{p f}^{1}\left(G_{v}, H_{v} ; U_{n}^{e t}\right):=r_{v}^{-1}\left(H_{f}^{1}\left(H_{v}, U_{n}^{e t}\right)\right) .
$$

We know that $E$ acquires good reduction everywhere after base extension to the field $F$ generated by the 12-torsion ([29] p. 498, Corollary 3 and section 4). We then take the $H_{v}$ to be the Galois groups of the completions $F_{v}$ for each place $v$ of $F$ lying above a place of $T$. Finally, we define

$$
H_{p f}^{1}\left(G_{p}, U_{n}^{e t}\right):=\cap_{v \mid p} H_{p f}^{1}\left(G_{p}, H_{v}, U_{n}^{e t}\right)
$$

and

$$
H_{\text {pun }}^{1}\left(G_{v}, U_{n}^{e t}\right):=\cap_{w \mid v} H_{p u n}^{1}\left(G_{v}, H_{w}, U_{n}^{e t}\right),
$$

for $v \neq p$. Then the global group $\operatorname{Sel}_{T}\left(U_{n}\right)$ is defined to be the intersection of the inverse images of all these local groups under the global-to-local restriction maps:

$$
\operatorname{Sel}_{T}\left(U_{n}\right):=\cap_{v \in T}\left[\left(\operatorname{Res}_{l o c, v}^{\text {glob }}\right)^{-1}\left(H_{p u n / p f}^{1}\left(G_{v}, U_{n}^{e t}\right)\right)\right] \subset H^{1}\left(G_{T}, U_{n}^{e t}\right) .
$$

With these definitions in place we claim there is an exact sequence

$$
0 \rightarrow \operatorname{Sel}_{T}\left(Z^{n+1} \backslash Z^{n}\right) \rightarrow \operatorname{Sel}_{T}\left(G_{T}, U_{n+1}^{e t}\right) \rightarrow \operatorname{Sel}_{T}\left(U_{n}^{e t}\right)
$$

again in the sense that the vector group on the left acts freely on the middle with quotient a subvariety of the right hand side. Most of the proof is a consequence of the exactness of

$$
0 \rightarrow H^{1}\left(G_{T}, Z^{n+1} \backslash Z^{n}\right) \rightarrow H^{1}\left(G_{T}, U_{n+1}^{e t}\right) \rightarrow H^{1}\left(G_{T}, U_{n}^{e t}\right)
$$

([23], section 1) leaving us to check that if $c_{1}, c_{2}$ are two elements of $\operatorname{Sel}_{T}\left(G_{T}\right.$, $\left.U_{n+1}^{e t}\right)$ mapping to the same class in $\operatorname{Sel}_{T}\left(G_{T}, U_{n}^{e t}\right)$, then one is a translate of the other by a class in $\operatorname{Sel}_{T}\left(Z^{n+1} \backslash Z^{n}\right)$. This can be checked locally at each prime. So let $w$ be a prime of $F$ lying above $p$ and let $c_{1}, c_{2} \in Z^{1}\left(H_{w}, U_{n+1}^{e t}\right)$ be two cocycles representing classes of $H_{f}^{1}\left(H_{w}, U_{n+1}^{e t}\right)$ and assume they have the same image in $H_{f}^{1}\left(H_{w}, U_{n}^{e t}\right)$. We already know that $c_{2}=c_{1} z$ for some cocycle $z \in Z^{1}\left(H_{w}, Z^{n+1} \backslash Z^{n}\right)$ and we need to check that it trivializes over $B_{c r}$. Since both $c_{1}$ and $c_{2}$ trivialize over $B_{c r}$, there are elements $u_{1}, u_{2} \in U_{n+1}^{e t}\left(B_{c r}\right)$ such that $c_{i}(g)=g\left(u_{i}^{-1}\right) u_{i}$ for $g \in H_{w}$. Then $z(g)=g\left(u_{1} u_{2}^{-1}\right) u_{2} u_{1}^{-1}$. Therefore, we 
see that $u_{2} u_{1}^{-1}$ maps to an $H_{w}$-invariant element in $U_{n}^{e t}\left(B_{c r}\right)$. But the invariant points just comprise $U_{n}^{D R}$ and the map $U_{n+1}^{D R} \rightarrow U_{n}^{D R}$ is surjective. Therefore, there is an $H_{w}$-invariant element $v \in U_{n+1}\left(B_{c r}\right)$ such that $u_{2} u_{1}^{-1}=v c$ for some $c \in Z^{n+1} \backslash Z^{n}\left(B_{c r}\right)$. Hence, $z(g)=g\left((v c)^{-1}\right)(v c)=g\left(c^{-1}\right) c$, giving us the triviality we want. (Of course we should have included a $\mathbb{Q}_{p}$-algebra $R$ in the notation to prove all this functorially, but we have omitted it for brevity.) At a prime $w$ not dividing $p$, the condition to check concerns unramified cocycles, and hence is easier: Suppose $c_{1}, c_{2}$ are two unramified cocycles with values in $U_{n+1}^{e t}$ that map to the same class in $H^{1}\left(H_{w}, U_{n}^{e t}\right)$. Then $c_{2}=c_{1} z$ for some cocycle $z$ with values in $Z^{n+1} \backslash Z^{n}$. But since the action on $U_{n+1}^{e t}$ itself is unramified, all cocycles restricted to the inertia group $I_{w}$ are homomorphisms. Then since $c_{1}$ and $c_{2}$ are in fact trivial on $I_{w}$, so is $z$.

For $n=2$, we get from this the inequality of dimensions

$$
\operatorname{dim} \operatorname{Sel}_{T}\left(U_{3}^{e t}\right) \leq \operatorname{dim} S e l_{T}\left(U_{2}^{e t}\right)+\operatorname{dimSel}_{T}\left(Z^{3} \backslash Z^{2}\right) .
$$

However,

$$
Z^{3} \backslash Z^{2} \simeq H_{1}\left(\bar{E}, \mathbb{Q}_{p}\right) \wedge H_{1}\left(\bar{E}, \mathbb{Q}_{p}\right) \simeq \mathbb{Q}_{p}(1) .
$$

This implies that $\operatorname{Sel}_{T}\left(Z^{3} \backslash Z^{2}\right)$ consists of the units in $\mathbb{Z}$ tensor $\mathbb{Q}_{p}$, and hence, is zero ([3], example 3.9). Therefore,

$$
\operatorname{dim} \operatorname{Sel}_{T}\left(U_{3}^{e t}\right)=\operatorname{dim} \operatorname{Sel}_{T}\left(U_{2}^{e t}\right)=\operatorname{dim} \operatorname{Sel}_{T}\left(H_{1}\left(\bar{E}, \mathbb{Q}_{p}\right)\right)=1,
$$

by the rank one hypothesis.

On the De Rham side, we have the same dimension count (=1) for $Z^{3} \backslash Z^{2}$. Meanwhile, considering the quotient map

$$
H_{1}^{D R}\left(E_{p}\right) \otimes H_{1}^{D R}\left(E_{p}\right) \rightarrow Z^{3} \backslash Z^{2}
$$

and the fact that $F^{0} H_{1} \wedge F^{0} H_{1}=0$, we get $F^{0}\left(Z^{3} \backslash Z^{2}\right)=0$. So

$$
\operatorname{dim} F^{0} U_{3}^{D R}=\operatorname{dim} F^{0} U_{2}^{D R}=1
$$

and

$$
\operatorname{dim} U_{3}^{D R} / F^{0}=3-1=2 .
$$

Therefore, $j_{3}^{D R}$ suffices to finish the job.

This calculation shows that in explicit situations like this with a fixed $n$, it is worth imposing extra Selmer conditions to improve the dimension estimate, in contrast to the general finiteness problem where refined conditions appear to be irrelevant. It is probably also worth looking into this case more carefully 
with a view towards working out explicit bounds in the manner of Coleman's refinement of Chabauty [7]. The suspicion is that this will require understanding the Dieudonné map $D$ more precisely, along the lines of 'non-abelian explicit reciprocity laws' à là Kato [22].

It was pointed out to me by John Coates that the general CM case is intimately related to a pseudo-nullity conjecture from the Iwasawa theory of elliptic curves. That is, let $F_{\infty}$ be the field generated by the $p$-power torsion of $E, F_{1}$ the field generated by the $p$-torsion, $G_{\infty}=\operatorname{Gal}\left(F_{\infty} / F_{1}\right)$, and $\Lambda=$ $\mathbb{Z}_{p}\left[\left[G_{\infty}\right]\right]$ the Iwasawa algebra of $G_{\infty}$. Let $I$ be the Galois group of the maximal abelian unramified pro-p extension of $F_{\infty}$ split over the primes lying above $T$. Coates and Sujatha have conjectured [9] that $I$ is pseudo-null as a $\Lambda$-module.

Observation 4. This pseudo-nullity implies conjecture 1 for $E \backslash\{0\}$.

Recall that what is necessary is to estimate $H^{2}\left(G_{T}, Z^{n+1} \backslash Z^{n}\right)$ which is implied by an estimate for $H^{2}\left(G_{T}, H_{1}\left(\bar{E}, \mathbb{Q}_{p}\right)^{\otimes n}\right)$. But for just this part, we can assume that the base-field is $F_{1}$ since the corestriction map is surjective on $H^{2}$. In fact, the pseudo-nullity implies that

$$
\operatorname{dim} H^{2}\left(G_{T}, H_{1}\left(\bar{E}, \mathbb{Q}_{p}\right)^{\otimes n}\right) \leq P(n)
$$

for some polynomial $P(n)$. To see this, first note that it suffices to prove such an inequality for the Selmer group

$$
\operatorname{Ker}\left(H^{2}\left(G_{T}, H_{1}\left(\bar{E}, \mathbb{Q}_{p}\right)^{\otimes n}\right) \rightarrow \oplus_{v \in T} H^{2}\left(G_{v}, H_{1}\left(\bar{E}, \mathbb{Q}_{p}\right)^{\otimes n}\right)\right)
$$

since the local contributions can be bounded exactly as in section 3 (note that $g=1$ in this case). So, Poitou-Tate duality, it suffices to bound the $H^{1}$ Selmer group

$$
\begin{aligned}
\operatorname{Sel}_{T}^{0}\left(H^{1}\left(\bar{E}, \mathbb{Q}_{p}\right)^{\otimes n}(1)\right)= & \operatorname{Ker}\left(H^{1}\left(G_{T}, H^{1}\left(\bar{E}, \mathbb{Q}_{p}\right)^{\otimes n}(1)\right)\right. \\
& \left.\rightarrow \oplus_{v \in T} H^{1}\left(G_{v}, H^{1}\left(\bar{E}, \mathbb{Q}_{p}\right)^{\otimes n}(1)\right)\right),
\end{aligned}
$$

and we have an isomorphism:

$$
\left.\operatorname{Sel}_{T}^{0}\left(H^{1}\left(\bar{E}, \mathbb{Q}_{p}\right)^{\otimes n}(1)\right) \simeq \operatorname{Hom}_{\Lambda}\left(I, H^{1}\left(\bar{E}, \mathbb{Q}_{p}\right)^{\otimes n}\right)(1)\right)
$$

coming from the Hochschild-Serre spectral sequence. To see this, it is perhaps convenient to utilize the language of continuous étale cohomology [21] applied to the inverse system

$$
\left(T_{i}\right)=\left(H^{1}\left(\bar{E}, \mathbb{Z} / p^{i}\right)^{\otimes n}(1)\right) .
$$


The Hochschild-Serre spectral sequence in this context gives us the exact sequence

$$
\begin{aligned}
0 & \rightarrow H^{1}\left(G_{\infty},\left(H^{0}\left(N, T_{i}\right)\right)\right) \rightarrow H^{1}\left(G_{T},\left(T_{i}\right)\right) \\
& \rightarrow H^{0}\left(G_{\infty},\left(H^{1}\left(N, T_{i}\right)\right)\right) \rightarrow H^{2}\left(G_{\infty},\left(H^{0}\left(N, T_{i}\right)\right)\right)
\end{aligned}
$$

([21], theorem 3.3), where $N \subset G_{T}$ is the subgroup fixing $F_{\infty}$. Since the $N$-action is trivial, all the $H^{0}\left(N, T_{i}\right)$ are just $T_{i}$, and hence, satisfy the MittagLeffler condition. Therefore, all the terms but one become readily identified with the continuous Galois cohomology of $T=\lim _{\longleftarrow} T_{i}=H^{1}\left(\bar{E}, \mathbb{Z}_{p}\right)^{\otimes n}(1)$ while

$$
H^{0}\left(G_{\infty},\left(H^{1}\left(N, T_{i}\right)\right)\right):=\lim _{\longleftarrow} \operatorname{Hom}_{G_{\infty}}\left(N, T_{i}\right)=\operatorname{Hom}_{G_{\infty}}(N, T) .
$$

When we tensor with $\mathbb{Q}$, we get therefore

$$
H^{1}\left(G_{T}, H^{1}\left(\bar{E}, \mathbb{Q}_{p}\right)^{\otimes n}(1)\right) \simeq \operatorname{Hom}_{\Lambda}\left(N, H^{1}\left(\bar{E}, \mathbb{Q}_{p}\right)^{\otimes n}(1)\right) .
$$

Passing to the Selmer group, the last group gets replaced by $\operatorname{Hom}_{\Lambda}(I$, $\left.H^{1}\left(\bar{E}, \mathbb{Q}_{p}\right)^{\otimes n}(1)\right)$.

But the pseudo-nullity implies that $I$ is finitely generated over $\mathbb{Z}_{p}$ (see, e.g., [15], section 2, paragraph following lemma 2). Thus, if we fix a Frobenius element $F$ in $G_{\infty}$, then only finitely many eigenvalues can occur for $F$ in $I \otimes \mathbb{Q}_{p}$. Weight considerations imply then that

$$
\operatorname{Hom}_{\Lambda}\left(I, H^{1}\left(\bar{E}, \mathbb{Q}_{p}\right)^{\otimes n}(1)\right)=0
$$

for $n>>0$.

\section{Acknowledgements}

The author was supported in part by a grant from the National Science Foundation. Deep gratitude is due to: A referee, for his thorough reading of the manuscript that gave rise to many valuable suggestions; Shinichi Mochizuki for overwhelming influence stemming from his 'non-linear' philosophy; Dick Hain for continuing to provide patient answers to questions on Hodge theory; Bill McCallum, for emphasizing over the years the importance of Chabauty's method; Dinesh Thakur, for a careful and enthusiastic reading of the manuscript; Martin Olsson, for communicating to me his deep results on non-abelian $p$-adic Hodge theory; Uwe Jannsen, Guido Kings, Jan Nekovar, and Tony Scholl, for helpful discussions on mixed motives; Gerd Faltings, Florian Pop, John Coates, Kazuya Kato, and Akio Tamagawa, for persistent encouragement of this research and many enlightening discussions on fundamental groups and Iwasawa theory; and, of course, Serge Lang, for too many things to be listed. 


\section{References}

[1] P. Berthelot, Finitude et pureté cohomologique en cohomologie rigide, Invent. Math. 128 (1997), no. 2, 329-377.

[2] A. Besser, Coleman integration using the Tannakian formalism, Math. Ann. 322 (2002), no. $1,19-48$.

[3] S. Bloch and K. Kato, $L$-functions and Tamagawa numbers of motives, in The Grothendieck Festschrift, Vol. I, 333-400, Progr. Math., 86, Birkhäuser, Boston, MA, 1990.

[4] C. Chabauty, Sur les points rationnels des courbes algébriques de genre supérieur à l'unité, C. R. Acad. Sci. Paris 212 (1941), 882-885.

[5] K. T. Chen, Iterated path integrals, Bull. Amer. Math. Soc. 83 (1977), no. 5, 831-879.

[6] B. Chiarellotto and B. Le Stum, F-isocristaux unipotents, Compositio Math. 116 (1999), no. $1,81-110$.

[7] R. F. Coleman, Effective Chabauty, Duke Math. J. 52 (1985), no. 3, 765-770.

[8] The monodromy pairing, Asian J. Math. 4 (2000), no. 2, 315-330.

[9] J. Coates and R. Sujatha, Fine Selmer groups of elliptic curves over $p$-adic Lie extensions, Math. Ann. 331 (2005), no. 4, 809-839.

[10] P. Deligne, Le groupe fondamental de la droite projective moins trois points, in Galois groups over $\mathbb{Q}$ (Berkeley, CA, 1987), 79-297, Springer, New York, 1989.

[11] G. Faltings, Mathematics around Kim's new proof of Siegel's theorem, Diophantine Goemetry, Proceedings of the research program at the Centro di Ricerca Matematica Ennio de Giorgi, U. Zannier (ed.), 390 pp., 2007.

[12] J.-M. Fontaine, Sur certains types de représentations $p$-adiques du groupe de Galois d'un corps local; construction d'un anneau de Barsotti-Tate, Ann. of Math. (2) 115 (1982), no. $3,529-577$.

[13] J.-M. Fontaine and B. Mazur, Geometric Galois representations, in Elliptic curves, modular forms, 83 Fermat's last theorem (Hong Kong, 1993), 41-78, Int. Press, Cambridge, MA, 1995.

[14] H. Furusho, $p$-adic multiple zeta values. I. $p$-adic multiple polylogarithms and the $p$-adic KZ equation, Invent. Math. 155 (2004), no. 2, 253-286.

[15] R. Greenberg, On the structure of certain Galois groups, Invent. Math. 47 (1978), no. 1, 85-99.

[16] A. Grothendieck, Brief an G. Faltings, in Geometric Galois actions, 1, 49-58, Cambridge Univ. Press, Cambridge, 1997.

[17] R. M. Hain, Higher Albanese manifolds, in Hodge theory (Sant Cugat, 1985), 84-91, Lecture Notes in Math., 1246, Springer, Berlin, 1987.

[18] The de Rham homotopy theory of complex algebraic varieties. I, $K$-Theory 1 (1987), no. 3, 271-324.

[19] R. M. Hain and S. Zucker, Unipotent variations of mixed Hodge structure, Invent. Math. 88 (1987), no. $1,83-124$.

[20] U. Jannsen, On the $l$-adic cohomology of varieties over number fields and its Galois cohomology, in Galois groups over $\mathbb{Q}$ (Berkeley, CA, 1987), 315-360, Springer, New York, 1989

[21] Continuous étale cohomology, Math. Ann. 280 (1988), no. 2, 207-245.

[22] K. Kato, $p$-adic Hodge theory and values of zeta functions of modular forms, Astérisque No. 295 (2004), ix, 117-290.

[23] M. Kim, The motivic fundamental group of $\mathbf{P}^{1} \backslash\{0,1, \infty\}$ and the theorem of Siegel, Invent. Math. 161 (2005), no. 3, 629-656.

[24] J. P. Labute, On the descending central series of groups with a single defining relation, J. Algebra 14 (1970), 16-23.

[25] S. Lang, Division points on curves, Ann. Mat. Pura Appl. (4) 70 (1965), 229-234.

[26] J. Neukirch, A. Schmidt and K. Wingberg, Cohomology of number fields, Springer, Berlin, 2000. 
[27] M. Olsson, Towards non-abelian p-adic Hodge theory in the good reduction case, preprint. http://www.math.utexas.edu/ ${ }^{\sim}$ molsson. (Revised version, in preparation, to comprise the Hodge filtration.)

[28] A. Shiho, Crystalline fundamental groups and p-adic Hodge theory, in The arithmetic and geometry of algebraic cycles (Banff, AB, 1998), 381-398, Amer. Math. Soc., Providence, RI, 2000.

[29] J.-P. Serre and J. Tate, Good reduction of abelian varieties, Ann. of Math. (2) 88 (1968), $492-517$.

[30] V. Vologodsky, Hodge structure on the fundamental group and its application to $p$-adic integration, Mosc. Math. J. 3 (2003), no. 1, 205-247, 260.

[31] J. Wildeshaus, Realizations of polylogarithms, Lecture Notes in Math., 1650, Springer, Berlin, 1997.

[32] Z. Wojtkowiak, Cosimplicial objects in algebraic geometry, in Algebraic K-theory and algebraic topology (Lake Louise, AB, 1991), 287-327, Kluwer Acad. Publ., Dordrecht, 1993. 